\title{
Near Optimum Power Control and Precoding under Fairness Constraints in Network MIMO Systems
}

\author{
Gábor Fodor, ${ }^{1}$ Mikael Johansson, ${ }^{2}$ and Pablo Soldati ${ }^{2}$ \\ ${ }^{1}$ Ericsson Research, 16480 Stockholm, Sweden \\ ${ }^{2}$ Automatic Control Lab, Royal Institute of Technology, 10044 Stockholm, Sweden \\ Correspondence should be addressed to Gábor Fodor, gabor.fodor@ericsson.com
}

Received 1 July 2009; Accepted 25 October 2009

Academic Editor: Hongxiang Li

Copyright ( 2010 Gábor Fodor et al. This is an open access article distributed under the Creative Commons Attribution License, which permits unrestricted use, distribution, and reproduction in any medium, provided the original work is properly cited.

\begin{abstract}
We consider the problem of setting the uplink signal-to-noise-and-interference (SINR) target and allocating transmit powers for mobile stations in multicell spatial multiplexing wireless systems. Our aim is twofold: to evaluate the potential of such mechanisms in network multiple input multiple output (MIMO) systems, and to develop scalable numerical schemes that allow real-time nearoptimal resource allocation across multiple sites. We formulate two versions of the SINR target and power allocation problem: one for maximizing the sum rate subject to power constraints, and one for minimizing the total power needed to meet a sum-rate target. To evaluate the potential of our approach, we perform a semianalytical study in Mathematica using the augmented Lagrangian penalty function method. We find that the gain of the joint optimum SINR setting and power allocation may be significant depending on the degree of fairness that we impose. We develop a numerical technique, based on successive convexification, for real-time optimization of SINR targets and transmit powers. We benchmark our procedure against the globally optimal solution and demonstrate consistently strong performance in realistic network MIMO scenarios. Finally, we study the impact of near optimal precoding in a multicell MIMO environment and find that precoding helps to reduce the sum transmit power while meeting a capacity target.
\end{abstract}

\section{Introduction}

Recently, several works proposed and demonstrated various forms of tight network coordination as a means to provide high spectral efficiency in multicell multiple input multiple output (MIMO) cellular networks $[1,2]$. Such coordination among the cells deployed over a certain geographical area has initially aimed at coordinating transmitter and receiver algorithms [3-5]. These promising results have triggered the interest of standardization bodies and industry players to investigate the architecture and protocol aspects of network MIMO systems employing multisite coordination of signal transmission and reception $[6,7]$.

Since network MIMO systems in general and multicell spatial multiplexing systems in particular inherently support the exchange of control information among multiple base stations, they can readily benefit of joint radio resource management functions, such as multicell scheduling, power control and precoding [8-10]. Multicell scheduling is concerned with assigning radio resources to users in multiple cells such that some utility function is maximized; see, for instance, $[11,12]$. Multicell power control can be viewed as a finer granularity control which is concerned with allocating power to scheduled users. Specifically for the uplink, it has been shown that coordinated power control can minimize the overall transmit power so as to maintain a predetermined signal-to-noise-and-interference (SINR) target [13].

For multicell scenarios, Hande et al. have demonstrated significant advantages of optimizing the SINR targets according to some criterion set by the network operator [14]. That work used SINR expressions for single input single output (SISO) systems without spatial multiplexing and considered network utility maximization problems with fair user utility functions $u_{i}(\cdot)$, in the sense that if the SINR tends to zero, $u_{i} \rightarrow-\infty$. If proportionally fair rate allocations are desired, the SINR targets could also be set via optimal 
distributed power control algorithms; see, for example, [15, 16]. However, none of these methods are easily extended to throughput maximization problems.

Similarly, we expect that setting the SINR targets in multiple network MIMO cells is an efficient means to control fairness and multicell throughput performance. Unlike traditional cellular networks, network MIMO systems allow the adjustment of these SINR targets on a time scale that is similar to scheduling and power control. Roughly speaking, manipulating the SINR targets can be seen as an extension of network MIMO power control algorithms (in the spirit of [14]) that adjust the individual power levels taking into account the channel variations such that predetermined SINR targets are reached and the overall power is minimized.

Building on this key observation, the first contribution of this paper is to evaluate the potential of such mechanisms in multicell spatial multiplexing systems. To this end, we develop a model that jointly optimizes the SINR targets and the power levels for the uplink of spatially multiplexing network MIMO systems. Our model can explicitly take into account fairness constraints by requiring that the ratio of the individual SINR (and thereby rate) targets for different mobile stations remain under some prescribed value, ranging from greedy throughput maximization ("no fairness") to equal rate allocation. Secondly, we develop scalable numerical schemes that allow real-time near-optimal resource allocation across multiple sites. Our major finding is that the new degree of freedom in network MIMO systems (i.e., multicell SINR target control) is an efficient tool to control the throughput performance and fairness in multicell systems. Finally, we find that when mobile stations employ fast, channel aware precoding, they can either significantly reduce their transmission power while maintaining a capacity target or enhance the throughput for a fixed power budget.

The paper is structured as follows. Section 2 models the uplink transmission of a network MIMO system employing minimum mean square error (MMSE) receiver. Section 3 formulates the SINR setting and power allocation problem. Sections 4 and 5 present a semianalytical and numerical solution approaches, respectively. Section 7 discusses numerical results and Section 8 concludes the paper.

\section{System Model}

2.1. Modelling the Received Signal. In order to establish the received signal model, we revise and merge the models of [13, $17,18]$. We consider the uplink transmission of a multicell system with $K$ cells and assume that each cell consists of a base station (BS) with $N_{r}$ being receive antennas and an active mobile station (MS) with $N_{t}$ being transmit antennas and spatial multiplexing. The assumption of having a single MS in each cell is not limiting, because it includes time division, (orthogonal) frequency division and orthogonal code division, multiplexing systems which ensure (time, frequency, or code domain) orthogonality within a single cell [13]. A narrow-band quasistatic flat-fading channel is assumed, where the channel remains constant within several scheduling instances (frames).
The received signal at the $k$ th BS is represented as

$$
\mathbf{y}_{k}=\alpha_{k, k} \mathbf{H}_{k, k} \mathbf{T}_{k} \mathbf{x}_{k}+\sum_{j \neq k} \alpha_{k, j} \mathbf{H}_{k, j} \mathbf{T}_{j} \mathbf{x}_{j}+\mathbf{n}_{k},
$$

where

(i) $\alpha_{k, j}=\sqrt{P_{j} d_{k, j}^{-\rho} \chi_{k, j} / N_{t}}$ is a scalar coefficient depending on the total transmit power $P_{j}$ for user $j$, the lognormal shadow fading $\chi_{k, j}$, and distance $d_{k, j}$ between the $k$ th base station and the $j$ th user with path loss exponent $\rho$;

(ii) $\mathbf{x}_{k} \in \mathbb{C}^{N_{t} \times 1}$ is the data vector that is assumed to be zero-mean, normalized, and uncorrelated, $\mathbb{E}\left(\mathbf{x}_{k} \mathbf{x}_{k}^{\dagger}\right)=$ $\mathbf{I}_{N_{t}}$;

(iii) $\mathbf{H}_{k, j}$ denotes the $\left(N_{r} \times N_{t}\right)$ channel transfer matrix;

(iv) $\mathbf{T}_{k}$ is the MS- $k\left(N_{t} \times N_{t}\right)$ diagonal power loading matrix; to keep the total transmit power constant, $\mathbf{T}_{k}$ must satisfy

$$
\mathbb{E}\left(\mathbf{T}_{k}^{\dagger} \mathbf{T}_{k}\right)=\operatorname{trace}\left(\mathbf{T}_{k} \mathbf{T}_{k}^{\dagger}\right)=\sum_{i=1}^{N_{t}}\left|\mathbf{T}_{k}^{(i, i)}\right|^{2}=N_{t} \quad \forall k ;
$$

(v) $\mathbf{n}_{k}$ is a $N_{r} \times 1$ additive white Gaussian noise vector at the $k$ th base station with zero mean and covariance matrix $\mathbf{R}_{n_{k}}=\mathbb{E}\left(\mathbf{n}_{k} \mathbf{n}_{k}^{\dagger}\right)=\sigma_{n}^{2} \mathbf{I}_{N_{r}}$ for all $k$.

We note that the underlying assumption of the last bullet item on equal noise covariance for all base stations is reasonable for a set of base stations with the same antenna configuration and other physical and hardware characteristics within a limited geographical region and is often used both in the literature and in standardization $[6,7,13,19]$.

We rewrite the signal model (1) in a compact form as

$$
\mathbf{y}_{k}=\alpha_{k, k} \mathbf{H}_{k, k} \mathbf{T}_{k} \mathbf{x}_{k}+\mathbf{z}_{k}+\mathbf{n}_{k},
$$

where $\mathbf{z}_{k}=\sum_{j \neq k} \alpha_{k, j} \mathbf{H}_{k, j} \mathbf{T}_{j} \mathbf{x}_{j}$ denotes the $\left(N_{r} \times 1\right)$ interference vector from users in other cells, with covariance matrix

$$
\mathbf{R}_{z_{k}}=\mathbb{E}\left(\mathbf{z}_{k} \mathbf{z}_{k}^{\dagger}\right)=\sum_{j \neq k} \alpha_{k, j}^{2} \mathbf{H}_{k, j} \mathbf{T}_{j} \mathbf{T}_{j}^{\dagger} \mathbf{H}_{k, j}^{\dagger} .
$$

For ease of notation, we define an equivalent noise vector that accounts both intercell interference and background noise

$$
\mathbf{v}_{k}=\mathbf{z}_{k}+\mathbf{n}_{k}
$$

It is easy to show that $\mathbf{v}_{k}$ is zero-mean with covariance $\mathbf{R}_{v_{k}}=$ $\mathbf{R}_{z_{k}}+\mathbf{R}_{n_{k}}$; see Appendix A.

2.2. MMSE Receiver Error Matrix and the Effective SINR. As we shall see, calculating the error matrix of the specific receiver that we employ in our system is a prerequisite for calculating the SINR. In this work we assume that the received signal is filtered through a linear MMSE receiver with weighting matrix $\mathbf{G}_{k}$ to obtain the following estimate:

$$
\widehat{\mathbf{x}}_{k}=\mathbf{G}_{k} \mathbf{y}_{k}
$$


Proposition 1. For the linear MMSE receiver

$$
\begin{aligned}
\mathbf{G}_{k}= & \frac{1}{\alpha_{k, k}} \mathbf{T}_{k}^{\dagger} \mathbf{H}_{k, k}^{\dagger} \\
& \times\left(\mathbf{H}_{k, k} \mathbf{T}_{k} \mathbf{T}_{k}^{\dagger} \mathbf{H}_{k, k}^{\dagger}+\sum_{j \neq k} \frac{\alpha_{k, j}^{2}}{\alpha_{k, k}^{2}} \mathbf{H}_{k, j} \mathbf{T}_{j} \mathbf{T}_{j}^{\dagger} \mathbf{H}_{k, j}^{\dagger}+\frac{\sigma_{n}^{2}}{\alpha_{k, k}^{2}} \mathbf{I}_{N_{r}}\right)^{-1} .
\end{aligned}
$$

In the special case of equal power distribution, that is, $\mathbf{T}_{k}=\mathbf{I}_{N_{t}}$, the MMSE weighting matrix becomes

$$
\mathbf{G}_{k}=\frac{1}{\alpha_{k, k}} \mathbf{H}_{k, k}^{\dagger}\left(\mathbf{H}_{k, k} \mathbf{H}_{k, k}^{\dagger}+\sum_{j \neq k} \frac{\alpha_{k, j}^{2}}{\alpha_{k, k}^{2}} \mathbf{H}_{k, j} \mathbf{H}_{k, j}^{\dagger}+\frac{\sigma_{n}^{2}}{\alpha_{k, k}^{2}} \mathbf{I}_{N_{r}}\right)^{-1} .
$$

Proof. See Appendix A.

The $\left(N_{t} \times N_{r}\right)$ linear MMSE weighting matrix $\mathbf{G}_{k}$ can be expressed in an alternative, more compact, form as

$$
\begin{aligned}
\mathbf{G}_{k} & =\frac{1}{\alpha_{k, k}} \mathbf{T}_{k}^{\dagger} \mathbf{H}_{k, k}^{\dagger}\left(\mathbf{H}_{k, k} \mathbf{T}_{k} \mathbf{T}_{k}^{\dagger} \mathbf{H}_{k, k}^{\dagger}+\frac{1}{\alpha_{k, k}^{2}} \mathbf{R}_{v_{k}}\right)^{-1} \\
& =\left(\mathbf{I}+\mathbf{T}_{k}^{\dagger} \mathbf{R}_{H_{k}} \mathbf{T}_{k}\right)^{-1} \alpha_{k, k} \mathbf{T}_{k}^{\dagger} \mathbf{H}_{k, k}^{\dagger} \mathbf{R}_{v_{k}}^{-1},
\end{aligned}
$$

where $\mathbf{R}_{H_{k}}=\alpha_{k, k}^{2} \mathbf{H}_{k, k}^{\dagger} \mathbf{R}_{v_{k}}^{-1} \mathbf{H}_{k, k}$; see, for example, [20, Chapter 12]. To derive the streamwise SINRs at base station $k$, we will need the diagonal elements of the error matrix of the MMSE filtered signal. To this end, the following proposition is useful.

Proposition 2. The MMSE estimation error matrix $\left(N_{r} \times N_{r}\right)$ for the kth base station is

$$
\mathbf{E}_{k}=\left(\alpha_{k, k} \mathbf{G}_{k} \mathbf{H}_{k, k} \mathbf{T}_{k}-\mathbf{I}\right)\left(\alpha_{k, k} \mathbf{T}_{k}^{\dagger} \mathbf{H}_{k, k}^{\dagger} \mathbf{G}_{k}^{\dagger}-\mathbf{I}\right)+\mathbf{G}_{k} \mathbf{R}_{v_{k}} \mathbf{G}_{k}^{\dagger},
$$

or, equivalently

$$
\mathbf{E}_{k}=\left(\mathbf{I}+\mathbf{T}_{k}^{\dagger} \mathbf{R}_{H_{k}} \mathbf{T}_{k}\right)^{-1}
$$

Proof. The computation is derived in Appendix A.

Note that with equal power distribution, that is, $\mathbf{T}_{k}=\mathbf{I}_{N_{t}}$ these results reduce to [13, Appendix A].

We are now in the position to calculate the SINR for the signal model (3) assuming a linear MMSE receiver. Using the linear MMSE weighting matrix $\mathbf{G}_{k}$, the MSE and SINR expressions can be rewritten, respectively, as

$$
\begin{aligned}
& \operatorname{MSE}_{k, s} \triangleq\left(\mathbf{E}_{k}\right)_{(s, s)}=\left\{\left(I+\mathbf{T}_{k}^{\dagger} \mathbf{R}_{H_{k}} \mathbf{T}_{k}^{\dagger}\right)^{-1}\right\}_{(s, s)}, \\
& \gamma_{k, s} \triangleq \frac{1}{\mathrm{MSE}_{k, s}}-1 .
\end{aligned}
$$

2.3. Summary. In this section we defined the multicell MIMO-received signal model (3) and, assuming a linear MMSE receiver, derived the associated effective $\operatorname{SINR}\left(\gamma_{k, s}\right)$ for each stream of the received signal. Equations (12) are important because they capture the dependence of the SINRs on the transmission powers of the own MS and the interfering MSs through the $\mathbf{R}_{H_{k}}$ 's and the $\mathbf{R}_{v_{k}}$ 's. Thus, these relations serve as the basis for the optimization problems of the next section.

\section{Problem Formulation}

Our aim is to develop a mathematical framework for systematic optimization of SINR-targets, transmit powers, and precoding matrix to maximize a rate objective subject to power budget and fairness constraints (or to minimize power subject to rate constraints). To the best of the authors' knowledge, there are not efficient means for jointly optimizing all these variables. We build our theoretical developments on the following result from [13]: by assuming equal power allocation for all streams $s$ (i.e., no uplink beam forming, $\mathbf{T}_{k}=\mathbf{I}_{N_{t}}$ for all $k$ ), the minimum stream SINR is lower bounded as

$$
\min _{s \in\left[1, N_{t}\right]} \gamma_{k, s} \geq \underline{\gamma}_{k}(\mathbf{p})
$$

where $\mathbf{p}=\left(P_{1} \cdots P_{K}\right)^{T}$ is the power allocation vector, and

$$
\underline{\gamma}_{k}(\mathbf{p})=\frac{P_{k} d_{k, k}^{-\rho} \chi_{k, k}}{\sum_{j \neq k} P_{j} d_{k, j}^{-\rho} \chi_{k, j} \mu_{\max }\left(\boldsymbol{\Omega}_{k, j, 1}\right)+N_{t} \sigma_{k}^{2} \mu_{\max }\left(\boldsymbol{\Omega}_{k, j, 2}\right)} .
$$

Here, $\mu_{\max }(\cdot)$ is the maximum eigenvalue operator for a Hermitian matrix, while $\boldsymbol{\Omega}_{k, j, 1}$ and $\boldsymbol{\Omega}_{k, j, 2}$ are defined as

$$
\begin{aligned}
& \boldsymbol{\Omega}_{k, j, 1}=\left(\mathbf{H}_{k, k}^{\dagger} \mathbf{H}_{k, k}\right)^{-1} \mathbf{H}_{k, k}^{\dagger} \mathbf{H}_{k, j} \mathbf{H}_{k, j}^{\dagger} \mathbf{H}_{k, k}\left(\mathbf{H}_{k, k}^{\dagger} \mathbf{H}_{k, k}\right)^{-1}, \\
& \boldsymbol{\Omega}_{k, j, 2}=\left(\mathbf{H}_{k, k}^{\dagger} \mathbf{H}_{k, k}\right)^{-1} .
\end{aligned}
$$

This bound allows to associate a single SINR value

$$
\gamma_{k}(\mathbf{p}) \triangleq \min _{s \in\left[1, N_{t}\right]} \gamma_{k, s}
$$

with each MS- $k$. In what follows, we will search for SINR targets $\gamma_{k}^{\text {tgt }}$ which are feasible for the lower-bound (and hence for each individual stream) and let $\Gamma=\operatorname{diag}\left(\gamma_{1}^{\mathrm{tgt}} \cdots \gamma_{K}^{\mathrm{tgt}}\right)$.

3.1. Minimizing Sum Power under Fixed SINR Target. The above result was used in [13] to design power control schemes which maintain a fixed minimum SINR target $\gamma_{k}^{\text {tgt }}$ for every stream $s$ by enforcing $\gamma_{k}(\mathbf{p}) \geq \gamma_{k}^{\text {tgt }}$ for each user. As shown in [13], the transmit power of MS- $k$ must satisfy

$$
\begin{gathered}
P_{k} \geq \gamma_{k}^{\mathrm{tgt}} \cdot\left(\frac{\sum_{j \neq k} P_{j} \cdot d_{k, j}^{-\rho} \chi_{k, j} \mu_{\max }\left(\boldsymbol{\Omega}_{k, j, 1}\right)}{d_{k, k}^{-\rho} \chi_{k, k}}\right. \\
\left.+\frac{\sigma_{n}^{2} N_{t} \mu_{\max }\left(\boldsymbol{\Omega}_{k, j, 2}\right)}{d_{k, k}^{-\rho} \chi_{k, k}}\right)
\end{gathered}
$$


Moreover, the power vector that satisfies this requirement and minimizes the sum power is

$$
\mathbf{p}^{\star}=(\mathbf{I}-\mathbf{\Gamma F})^{-1} \mathbf{\Gamma n},
$$

where $\mathbf{n}$ is a $K$-dimensional effective noise variance vector whose $k$ th element is $n_{k}=N_{t} \sigma_{n}^{2} \mu_{\max }\left(\boldsymbol{\Omega}_{k, j, 2}\right) / d_{k, k}^{-\rho} \chi_{k, k}$, and

$$
\mathbf{F}_{k, j}= \begin{cases}\frac{d_{k, j}^{-\rho} \chi_{k, j} \mu_{\max }\left(\boldsymbol{\Omega}_{k, j, 1}\right)}{d_{k, k}^{-\rho} \chi_{k, k}}, & k \neq j, \\ 0, & k=j .\end{cases}
$$

Still, (18) requires feasibility of the SINR targets, which in practice cannot be guaranteed a priori. Precoding optimization was shown to be effective to balance the conservativeness of the bound (13) and increase feasibility; see [13].

3.2. Optimal SINR Target Selection. In this paper we take a step further and explore a key observation, not fully exploited in [13]. Since the minimum user-stream SINR bound (13) allows to associate a single SINR target per user, one can regard each MS-BS connection as an equivalent SISO system and model the minimum user-stream capacity as function of the power allocation with a Shannon-like expression (normalized to the bandwidth) as

$$
c_{k}\left(\gamma_{k}^{\mathrm{tgt}}\right)=\log _{2}\left(1+\gamma_{k}^{\mathrm{tgt}}\right) \quad \forall k
$$

where we enforce

$$
\underline{\gamma}_{k}(\mathbf{p})=\frac{P_{k}}{n_{k}+\sum_{j \neq k} G_{k j} P_{j}} \geq \gamma_{k}^{\mathrm{tgt}} \quad \forall k
$$

with $\mathbf{G}=\mathbf{I}+\mathbf{F}$. This observation is the basis for optimizing the minimum user-stream SINR targets.

In network MIMO, it is possible (and as we shall see beneficial) to exploit the possibility to set the SINR targets such that the sum power is kept at a minimum level and the overall system capacity (sum rate) target $c_{\mathrm{m}}$ is reached. This problem is formulated as follows:

$$
\begin{array}{ll}
\underset{\Gamma, \mathbf{p}}{\operatorname{minimize}} & \sum_{k} P_{k} \\
\text { subject to } & \sum_{k} c_{k}\left(\gamma_{k}^{\mathrm{tgt}}\right) \geq c_{\mathrm{m}} \\
& \gamma_{k}^{\mathrm{tgt}}=\underline{\gamma}_{k}(\mathbf{p}) \quad \forall k,
\end{array}
$$

in the optimization variables $\boldsymbol{\Gamma}$ (SINR targets) and $\mathbf{p}$ (power). We are also interested in the dual formulation of problem (22), that is, maximizing the multicell capacity (sum rate) subject to a total power budget:

$$
\begin{array}{ll}
\underset{\Gamma, \mathbf{p}}{\operatorname{maximize}} & \sum_{k} c_{k}\left(\gamma_{k}^{\mathrm{tgt}}\right) \\
\text { subject to } & \sum_{k} P_{k} \leq P_{\mathrm{tot}} \\
& \gamma_{k}^{\mathrm{tgt}}=\underline{\gamma}_{k}(\mathbf{p}) \quad \forall k .
\end{array}
$$

3.3. Enforcing Fairness Constraints. Fairness can be enforced in the above formulations by limiting the ratio between SINR targets, that is,

$$
\gamma_{k}^{\mathrm{tgt}} \leq \Phi_{k j} \gamma_{j}^{\mathrm{tgt}} \quad \forall k, j \neq k
$$

The matrix $\Phi$ collects the fairness ratios. These constraints are written more compactly as $\mathbf{a}(\boldsymbol{\Gamma}) \preceq \mathbf{b}(\boldsymbol{\Gamma})$, where $\mathbf{a}=\operatorname{vec}\left(\mathbf{a}_{1} \cdots \mathbf{a}_{k}\right)$ with $\mathbf{a}_{k}=\left(\mathbf{1}-\mathbf{e}_{k}\right) \gamma_{k}^{\text {tgt }}$, and $\mathbf{b}(\boldsymbol{\Gamma})=$ $\operatorname{vec}(\boldsymbol{\Phi})$. (Here, $\mathbf{e}_{k}$ is the vector with 1 in the $k$ th coordinate and 0's elsewhere). To account for fairness constraints, we include the inequalities $\mathbf{a}(\boldsymbol{\Gamma}) \preceq \mathbf{b}(\boldsymbol{\Gamma})$ in (22) and (23). In what follows, we develop a novel efficient SINRtarget optimization procedure and combine it with iterative algorithms for power and precoding matrix optimization. As we will see, the minimum SINR bound (13) is quite conservative and including the precoding matrix $\mathbf{T}_{k}$ in the optimization is instrumental to enhance the performance.

\section{A Semianalytical Solution Approach}

We propose to solve the problems formulated in Section 3.2 through the augmented Lagrangian penalty function method [21]. In this method, the constrained nonlinear optimization task is transformed into an unconstrained problem by adding a penalty term to the Lagrangian function as follows:

$$
\begin{aligned}
L(\boldsymbol{\Gamma}, \mathbf{p}, \boldsymbol{\nu}, \mu, \varepsilon)= & \sum_{\mathrm{k}} P_{k}+\mu\left(\sum_{\mathrm{k}} c_{k}\left(\gamma_{k}^{\mathrm{tgt}}\right)-c_{\mathrm{m}}\right)+\boldsymbol{\nu}^{T}(\mathbf{a}-\mathbf{b}) \\
& +\varepsilon\left[\left(\sum_{\mathrm{k}} c_{k}\left(\gamma_{k}^{\mathrm{tgt}}\right)-c_{\mathrm{m}}\right)^{2}+\sum_{\mathrm{n}}\left(a_{n}-b_{n}\right)^{2}\right] .
\end{aligned}
$$

Here, we present the method for the power minimization problem (22). The Lagrangian for problem (23) follows similarly. It can be shown that if the optimum Lagrange multipliers are known, the solution to this unconstrained problem corresponds to the solution of the original problem (22) regardless of the value of the penalty parameter $\varepsilon$, see, for example, [21, Chapter 9].

4.1. Solution of the Power Minimization Problem. For ease of presentation, we consider a three cell system, that is $K=3$. First, we need to find the power vector as the function of the target multicell capacity (sum rate) $c_{\mathrm{m}}$ and the individual SINR targets (the $\gamma_{i}^{\text {tgt }}$ s):

$$
\mathbf{p}^{\star}\left(c_{\mathrm{m}}, \gamma_{1}^{\mathrm{tgt}}, \gamma_{2}^{\mathrm{tgt}}, \gamma_{3}^{\mathrm{tgt}}\right)=\left[\begin{array}{c}
\frac{M_{11}+M_{12}+M_{13}}{D_{p}} \\
\frac{M_{21}+M_{22}+M_{23}}{D_{p}} \\
\frac{M_{31}+M_{32}+M_{33}}{D_{p}}
\end{array}\right],
$$

where the parameters $M_{11}, \ldots, M_{33}$ and $D_{p}$ are given in Appendix B. From the capacity constraint, it follows that 
$(K-1)$ SINR values can be freely selected while the $K$ th SINR target value must be chosen such that the capacity constraint is fulfilled. In the case of $K=3$,

$$
\gamma_{3}^{\mathrm{tgt}}\left(c_{\mathrm{m}}, \gamma_{1}^{\mathrm{tgt}}, \gamma_{2}^{\mathrm{tgt}}\right)=e^{c_{\mathrm{m}}-\log \left(1+\gamma_{1}^{\mathrm{tgt}}\right)-\log \left(1+\gamma_{2}^{\mathrm{tgt}}\right)}-1
$$

Using this relationship, the $M_{i j}$ parameters are expressed as the functions of $\gamma_{1}^{\text {tgt }}$ and $\gamma_{2}^{\text {tgt }}$ (see Appendix B). That is, for a specific capacity target $c_{\mathrm{m}}, \mathrm{p}^{\star}$ and the sum of its components are expressed as a two-variable function of $\gamma_{1}^{\text {tgt }}$ and $\gamma_{2}^{\text {tgt }}$. Using (26), it is straightforward to find the stationary points of the unconstrained problem and, by establishing the second-order necessary conditions, to find the local optimum solutions (that is the local minimum points) of (22). In our Mathematica implementation, we found that in all considered practically relevant examples, a simple heuristic can then easily identify the global optimum solution (see also Section 7 ).

4.2. Solution of the Capacity Maximization Problem. For the capacity maximization case, we can freely choose $(K-1)$ SINR values, while the $K$ th SINR target needs to be selected to fulfil the constraint of (23). With an abuse of notation, let $P^{\star}=\sum_{i} P_{i}^{\star}$ denote the sum of the components of $\mathbf{p}^{\star}$ in (18). For the three cell system, summing the components of (18), it is straightforward to show that setting the SINR targets $\gamma_{1}^{\text {tgt }}$ and $\gamma_{2}^{\text {tgt }}$ implies setting $\gamma_{3}^{\text {tgt }}$ as follows:

$$
\begin{aligned}
\gamma_{3}^{\mathrm{tgt}}\left(P^{\star}, \gamma_{1}^{\mathrm{tgt}}, \gamma_{2}^{\mathrm{tgt}}\right) & \\
\leq & \frac{P^{\star}\left(1-F_{12} F_{21} \gamma_{1}^{\mathrm{tgt}} \gamma_{2}^{\mathrm{tgt}}\right)}{D_{c}} \\
& -\frac{\gamma_{1}^{\mathrm{tgt}} \gamma_{2}^{\mathrm{tgt}}\left(n_{1} F_{21}+n_{2} F_{12}\right)-n_{1} \gamma_{1}^{\mathrm{tgt}}-n_{2} \gamma_{2}^{\mathrm{tgt}}}{D_{c}}
\end{aligned}
$$

where $D_{c}$ is

$$
\begin{aligned}
D_{c}=P^{\star}\left(F_{13} F_{31} \gamma_{1}^{\mathrm{tgt}}+F_{23} F_{32} \gamma_{2}^{\mathrm{tgt}}+F_{12} F_{23} F_{31} \gamma_{1}^{\mathrm{tgt}} \gamma_{2}^{\mathrm{tgt}}\right. \\
\left.+F_{21} F_{13} F_{32} \gamma_{1}^{\mathrm{tgt}} \gamma_{2}^{\mathrm{tgt}}\right) \\
+\gamma_{1}^{\mathrm{tgt}} \gamma_{2}^{\mathrm{tgt}}\left(n_{1}\left(F_{23} F_{31}-F_{23} F_{32}+F_{21} F_{32}\right)\right. \\
+n_{2}\left(F_{13} F_{32}-F_{13} F_{31}+F_{12} F_{31}\right) \\
\left.+n_{3}\left(F_{13} F_{21}+F_{12} F_{23}-F_{12} F_{21}\right)\right) \\
+\gamma_{1}^{\mathrm{tgt}}\left(n_{1} F_{31}+n_{3} F_{13}\right)+\gamma_{2}^{\mathrm{tgt}}\left(n_{2} F_{32}+n_{3} F_{23}\right) .
\end{aligned}
$$

Similarly to the minimum $P^{\star}$ in problem (22), for a specific total power budget $P_{\text {tot }}$, (28) allows us to express the sum-rate $\sum c_{k}\left(\gamma_{k}^{\text {tgt }}\right)$ as a two-variable function of $\gamma_{1}^{\text {tgt }}$ and $\gamma_{2}^{\text {tgt }}$, which allows to derive the numerical results.

\section{Scalable Near-Optimal SINR Target Setting}

To design more scalable solutions and avoid the matrix inversion in (18), we make use of the model (20)-(21) to reformulate problem (23) as

$$
\begin{array}{ll}
\underset{\mathbf{p}, \mathbf{r}}{\operatorname{maximize}} & \sum_{k} r_{k} \\
\text { subject to } & r_{k} \leq c_{k}(\mathbf{p}) \quad \forall k \\
& \sum_{k} P_{k} \leq P_{\text {tot }}
\end{array}
$$

This problem optimizes the minimum user-stream transmission rates $\mathbf{r}$ and powers $\mathbf{p}$, hence implicitly the minimum user-stream SINR. Similarly to the formulation (23), problem (30) is not convex due to the link rate constraints $r_{k} \leq c_{k}(\mathbf{p})$.

5.1. Monotonic Optimization. Through an exponential transform of the variables $P_{k} \leftarrow e^{\widetilde{P}_{k}}$ and $r_{k} \leftarrow e^{\widetilde{r}_{k}}$ and a logtransformation of the constraints, we rewrite problem (30) as

$$
\begin{array}{ll}
\underset{\widetilde{\mathbf{p}}, \widetilde{\mathbf{r}}}{\operatorname{maximize}} & \sum_{k} e^{\widetilde{r}_{k}} \\
\text { subject to } & \widetilde{r}_{k} \leq \log \left(c_{k}\left(e^{\widetilde{\mathbf{p}}}\right)\right) \quad \forall k \\
& \sum_{k} e^{\widetilde{P}_{k}} \leq P_{\text {tot }} .
\end{array}
$$

Since the objective function is convex and monotonically increasing in the variables $\tilde{\mathbf{r}}$ and the feasibility set is convex, problem (31) falls into the family of monotonic optimization, for which, unlike standard convex optimization problems, local optimality does not translate into global optimality. Only recently, Qian et al. [22] have shown the equivalence between the formulations (30) and (31) and have devised an algorithm, MAPEL, that finds the global optimum solution by constructing a series of polyblocks that approximate the SINR region with increasing precision (see [22] for details).

Proposition 3. The MAPEL algorithm converges to the global optimal solution of problem (31).

Proof. It follows analogously to [22, Theorem 2] by defining the feasibility set as

$$
\mathcal{F}=\left\{\mathbf{p} \mid 0 \leq P_{k} \leq P_{\text {tot }}, \sum_{\mathrm{k}} P_{k} \leq P_{\text {tot }} f_{i}(\mathbf{p}) g_{i}(\mathbf{p})^{-1} \geq 1\right\},
$$

where $f_{i}(\mathbf{p})$ and $g_{i}(\mathbf{p})$ are defined as in [22]. In our case, we combine implicit peak-power constraints (i.e., $0 \leq P_{k} \leq P_{\text {tot }}$ ) with an explicit global power budget.

The MAPEL algorithm allows to trade-off between accuracy and convergence time by tuning an approximation factor $\delta$. Since the computation times drastically increase with 
increasing accuracy and problem size, MAPEL is currently not feasible for real-time SINR target setting. Nevertheless, it is an excellent candidate for off-line benchmarking of the low-complexity schemes that we will develop next.

5.2. An Approximation of the Link Rate Constraint. To reduce problem complexity, we use an approximation to "convexify" the problem. Inspired by $[15,23]$, we use the relation $\theta \log (x)+\beta \leq \log (1+x)$ with $\theta=x_{0} /\left(1+x_{0}\right)$ and $\beta=$ $\log \left(1+x_{0}\right)-\theta \log \left(x_{0}\right)$ to approximate the link capacity. The approximation becomes exact for $x=x_{0}$. We replace the expression (20) with a more conservative one:

$$
\tilde{c}_{k}(\mathbf{p})=\left[\theta_{k} \log _{2}\left(\underline{\gamma}_{k}(\mathbf{p})\right)+\beta_{k}\right] \leq c_{k}(\mathbf{p}) .
$$

By applying the approximation (33) to the stream rate constraints of problem (30), we obtain the following approximation of problem (30)

$$
\begin{array}{ll}
\underset{\Gamma, \mathbf{p}}{\operatorname{maximize}} & \sum_{k}\left[\theta_{k}^{(t)} \log _{2}\left(\gamma_{k}^{\mathrm{tgt}}\right)+\beta_{k}^{(t)}\right] \\
\text { subject to } & \gamma_{k}^{\mathrm{tgt}} \leq \frac{P_{k}}{n_{k}+\sum_{j \neq k} G_{k j} P_{j}} \quad \forall k \\
& \sum_{k} P_{k} \leq P_{\text {tot }},
\end{array}
$$

which explicitly optimizes the SINR targets $\Gamma$ and the transmit power p. Here, the SINR expression (21) has been added to the constraint set to provide an explicit relationship between these variables. Similarly to [15], we propose to solve problem (30) through a sequence of convex approximations according to the iterative Algorithm 1 . At the $t$ th iteration of the algorithm, the following problem $\mathcal{P}^{(t)}$ is solved:

$$
\begin{array}{ll}
\underset{\widetilde{\mathbf{\Gamma}}, \tilde{\mathbf{p}}}{\operatorname{maximize}} & \sum_{k}\left[\theta_{k}^{(t)} \widetilde{\gamma}_{k}^{\text {tgt }}+\beta_{k}^{(t)}\right] \\
\text { subject to } & \widetilde{\gamma}_{k}^{\text {tgt }} \leq \widetilde{P}_{k}-\log \left(n_{k}+\sum_{j \neq k} G_{k j} e^{\widetilde{P}_{j}}\right) \quad \forall k \\
& \sum_{k} e^{\widetilde{P}_{k}} \leq P_{\text {tot. }}
\end{array}
$$

The above formulation is obtained from problem (34) through the exponential change of variables $\gamma_{k}^{\text {tgt }} \leftarrow e^{\widetilde{\gamma}_{k}^{\text {tgt }}}$, $P_{k} \leftarrow e^{\widetilde{P}_{k}}$, and a log-transform of the constraints. Algorithm 1 iteratively solves the convex approximate problems $\left\{\mathcal{P}^{(t)}\right\}_{t}$ in the variables $\widetilde{\boldsymbol{\Gamma}}$ and $\widetilde{\mathbf{p}}$ and appropriately tunes $\theta$ and $\beta$ to improve the objective function until convergence.

While the approximation was initially proposed in [23] to tune the transmission power in DSL systems and then applied in [15] to network utility maximization problems with concave utilities, our formulation is used to optimize the SINR targets. We prove that each iteration of Algorithm 1 consists of a convex problem (Proposition 4) and that the sequence of solutions is convergent (Proposition 5), which follow quite straightforward from [15]. In addition, we
Initialize $\mathbf{p}^{(0)}, \theta^{(0)}$, and $\beta^{(0)}$ to some feasible values for the original problem (30).

Start with iteration step $t=1$.

(1) Solve the approximate optimization problem (35). Let $\left\{\boldsymbol{\Gamma}^{(t)}, \mathbf{p}^{(t)}\right\}$ denote the solution of the $t$ th iteration.

(2) Update $\boldsymbol{\theta}^{(t+1)}, \boldsymbol{\beta}^{(t+1)}$ at $\mathbf{x}_{0}^{(t)}=\gamma\left(\mathbf{p}^{(t)}\right)$.

(3) Update $t=t+1$ and repeat until convergence

Algorithm 1: Series of convex approximations.

demonstrate that the sequence converges to a solution that satisfies the KKT optimality condition of both the monotonic optimization (31) (Theorem 5.1) and the original nonconvex problem (30) (Theorem 5.2).

Proposition 4. The approximating problem $\mathcal{P}^{(t)}$ is convex.

Proof. The constraints contain a linear term in $\tilde{\gamma}_{k}$ and $\widetilde{P}_{k}$ and a convex term (log-sum-exp) in $\widetilde{\mathbf{p}}$. The power budget is convex (sum-exp), and the objective is linear in $\tilde{\gamma}_{k}$.

Proposition 5. The problem sequence $\left\{\mathcal{P}^{(t)}\right\}_{t}$ results in a series of monotonically improving objective values. The sequence always converges at which point the lower bound approximation (33) becomes exact.

Proof. The proof details can be found in Appendix C.

Theorem 5.1. The problem sequence $\left\{\mathcal{P}^{(t)}\right\}_{t}$ converges to a KKT-point of the monotonic optimization problem (31).

Proof. The proof follows from Proposition 5 and a direct inspection of the KKT optimality conditions for problems (C.1) and (31). The details can be found in Appendix C.

Theorem 5.2. The problem sequence $\left\{\mathcal{P}^{(t)}\right\}_{t}$ converges to a KKT-point of the original nonconvex problem (30).

Proof. See Appendix C.

\section{Precoding Optimization}

The mathematical framework devised in the previous sections allows to optimally select the SINR targets under equal power allocation for all streams (i.e., $\mathbf{T}_{k}=\mathbf{I}_{N_{t}}$ ) for two classes of problems: problem (22) minimizes the sum power while maintaining a fixed system capacity; problem (23) maximizes the multicell capacity subject to a fixed power budget. Both cases use the minimum per-stream SINR bound (13), that is,

$$
\gamma_{k}(\mathbf{p}) \geq \frac{P_{k} d_{k, k}^{-\rho} \chi_{k, k}}{\sum_{j \neq k} P_{j} d_{k, j}^{-\rho} \chi_{k, j} \mu_{\max }\left(\boldsymbol{\Omega}_{k, j, 1}\right)+N_{t} \sigma_{k}^{2} \mu_{\max }\left(\boldsymbol{\Omega}_{k, j, 2}\right)},
$$

to formulate the SINR and power allocation problem. Originally proposed in [13, Lemma 1], this bound applies 
Given $t=0, \epsilon^{(0)}=1, P_{\text {tot }}, \varepsilon_{\text {gap }}$ and $\mathbf{T}_{k}^{(0)}=\mathbf{I}_{N_{t}}$ for all $k$. Initialize SINR targets $\boldsymbol{\Gamma}^{(0)}=\operatorname{diag}\left(\gamma_{k}^{\mathrm{tgt}}\right)$ and transmission powers $\mathbf{p}^{(0)}$ solving either problem (22) or (23).

Repeat:

(1) $t=t+1$.

(2) For $k=1$ to $K$

(a) Given $\left\{\mathbf{T}^{(t-1)}, \mathbf{P}^{(t-1)}\right\}$, compute the inter ference $\zeta_{k, s}=f\left(\mathbf{T}^{(t-1)}, \mathbf{P}^{(t-1)}\right)$ as in (38).

(b) Calculate the optimum loading matrix $\mathbf{T}_{k}^{(t)}$ as

$$
\left(\mathbf{T}_{k}^{(t)}\right)^{(s, s)}=\sqrt{\frac{\zeta_{k, s} N_{t}}{\sum_{j=1}^{N_{t}} \zeta_{k, j}}} \quad \forall s \in\left[1, N_{t}\right] .
$$

(c) Calculate the new SINR targets $\boldsymbol{\Gamma}^{(t)}$ and update the optimum transmit power $P_{k}^{(t)}$ as

$$
\begin{aligned}
& \gamma_{k}^{(t)}=\gamma_{k}^{\mathrm{tgt}} \cdot \epsilon^{(t-1)} \quad \forall k \\
& P_{k}^{(t)}=\frac{\zeta_{k, s}}{\left|\left(\mathbf{T}_{k}^{(t)}\right)^{(s s)}\right|^{2}}\left(\gamma_{k}^{(t)}+1\right) \quad \forall k, s
\end{aligned}
$$

(3) Update the control parameter $\epsilon$ :

(a) If objective is power minimization: $\epsilon^{(t)}=\epsilon^{(t-1)}$;

(b) If objective is throughput maximization:

Until $\left|P_{k}^{(t)}-P_{k}^{(t-1)}\right| \leq \varepsilon_{\text {gap }}, \quad \forall k$.

$$
\epsilon^{(t)}=\left\{\epsilon^{(t-1)}-\kappa\left(\sum_{k} P_{k}^{(t)}-P_{\mathrm{tot}}\right)\right\}^{+} .
$$

Algorithm 2: Iterative SINR and precoding optimization.

to the minimum postprocessing user SINR with linear MMSE receiver with equal power allocation for all streams s. Unfortunately, the Rayleigh-Ritz Theorem [24] used in [13, Lemma 1] does not apply when the precoding matrix $\mathbf{T}_{k}$ is also included in the optimization framework. In what follows, we ask whether precoding optimization can bring an additional gain in network MIMO systems where the SINR targets are optimized based on this bound (without precoding).

From the signal model (1), when user $k$ th uses a diagonal power loading matrix $\mathbf{T}_{k} \in C^{N_{t} \times N_{t}}$ with $\sum_{s=1}^{N_{t}}\left|\mathbf{T}_{k}^{(s, s)}\right|^{2}=N_{t}$, the postprocessing SINR of its sth stream becomes

$$
\gamma_{k, s}=\frac{P_{k}\left|\mathbf{T}_{k}^{(s, s)}\right|^{2}}{\zeta_{k, s}}-1,
$$

where

$$
\begin{array}{r}
\zeta_{k, s}=\left\{\left(d _ { k , k } ^ { - \rho } \chi _ { k , k } \mathbf { H } _ { k , k } ^ { \dagger } \left(\sum_{j \neq k} P_{j} d_{k, j}^{-\rho} \chi_{k, j} \mathbf{H}_{k, j} \mathbf{T}_{j} \mathbf{T}_{j}^{\dagger} \mathbf{H}_{k, j}^{\dagger}\right.\right.\right. \\
\left.\left.\left.+N_{t} \sigma_{n}^{2} \mathbf{I}\right)^{-1} \mathbf{H}_{k, k}+\frac{1}{P_{k}} \mathbf{I}\right)^{-1}\right\}^{(s, s)}
\end{array}
$$

denotes the effective interference after MMSE processing. In [13], a heuristic algorithm for distributing the transmit power over different streams was presented. By inverting
(37) for fixed SINR targets, the algorithm finds a near optimal (sum power minimizing) precoding matrix for uplink transmission. Precoding optimization is shown to enhance the feasibility space of a rough SINR targets selection with respect to the equal power allocation case. By applying this algorithm to our optimized SINR targets for problem (22), the total sum power can be reduced further. However, some modifications are necessary for the capacity maximization problem. At the optimal point of problem (23), the SINR targets will consume the entire power budget $P_{\text {tot }}$. In this case, by better distributing the power budget $P_{\text {tot }}$, precoding optimization allows to sustain higher SINR targets, thus yielding a throughput gain.

To capitalize on these gains, we modify the algorithm in [13] as in Algorithm 2. The SINR targets are initialized to the optimal values $\Gamma=\operatorname{diag}\left(\gamma_{k}^{\text {tgt }}\right)$ yielded by either problem (22) or (23) without precoding, that is, with $\mathbf{T}_{k}=\mathbf{I}_{N_{t}}$ for all $k$. For sum-rate maximization, Algorithm 2 iteratively tunes the SINR targets, along with the precoding matrix and the transmission powers, until the entire power budget $P_{\text {tot }}$ is spent. At every iteration, the effective interference and the new precoding matrix are computed as in steps (a) and (b), respectively; the control parameter $\epsilon^{(t)}$ is used to update the SINR targets as

$$
\gamma_{k}^{(t)}=\gamma_{k}^{\mathrm{tgt}} \cdot \epsilon^{(t)} \quad \forall k
$$

which become the new reference for the power control update in step (c). Finally, $\epsilon^{(t)}$ is tuned differently for the two problems in step (3): for problem $(22), \epsilon^{(t)}$ is kept 


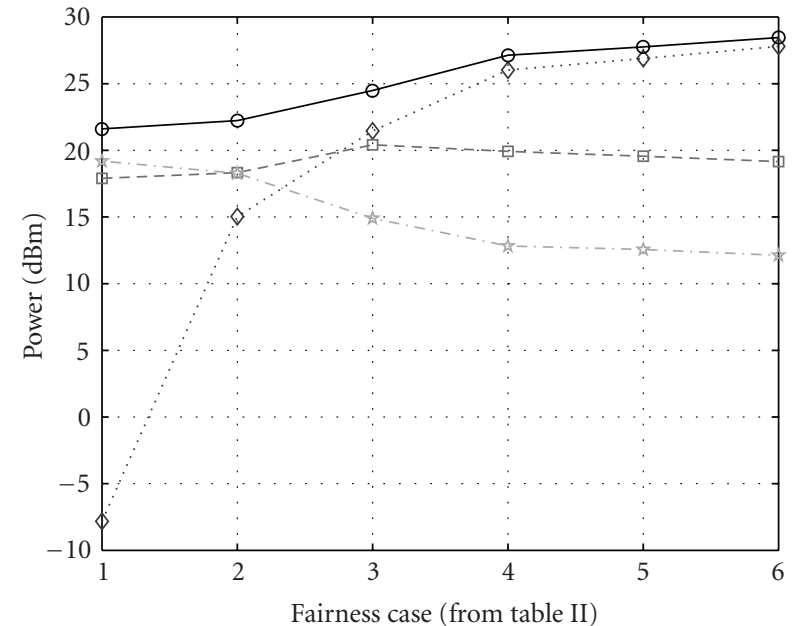

$$
\begin{array}{ll}
\therefore \Delta \cdot \text { User } 1 & \rightarrow-\text { User } 3 \\
-\boxminus-\text { User } 2 & - \text { - Sum power }
\end{array}
$$

(a) The individual power levels and the sum power for the six cases we study. The sum power is significantly lower $(21.6 \mathrm{dBm})$ without fairness than with fairness $(\approx 28.45 \mathrm{dBm})$. In fact, in a real system Cases 4-6 would hardly be feasible for User-3 due to the typical power limitation of MS $(\approx 24 \mathrm{dBm})$

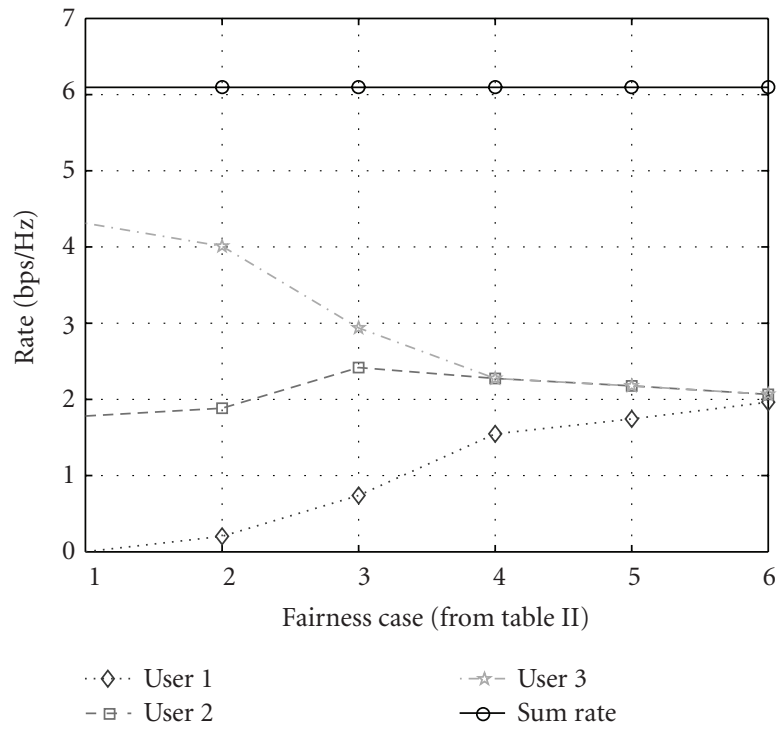

(b) The individual rates in the six fairness cases that we study. The sum rate is kept constant ( $2 \mathrm{bps} / \mathrm{Hz} / \mathrm{cell})$, but this sum rate is "distributed" unequally (Case 1) or nearly equally (Case 6) in the different cases

FIgURE 1: Sum power minimization subject capacity (sum rate) and fairness constraint.

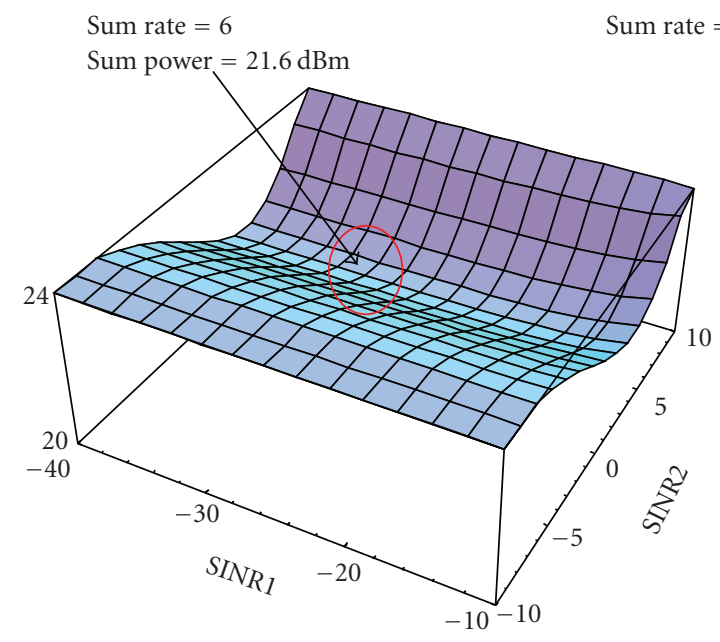

rate $=6$; antennas: $1 \times 2$
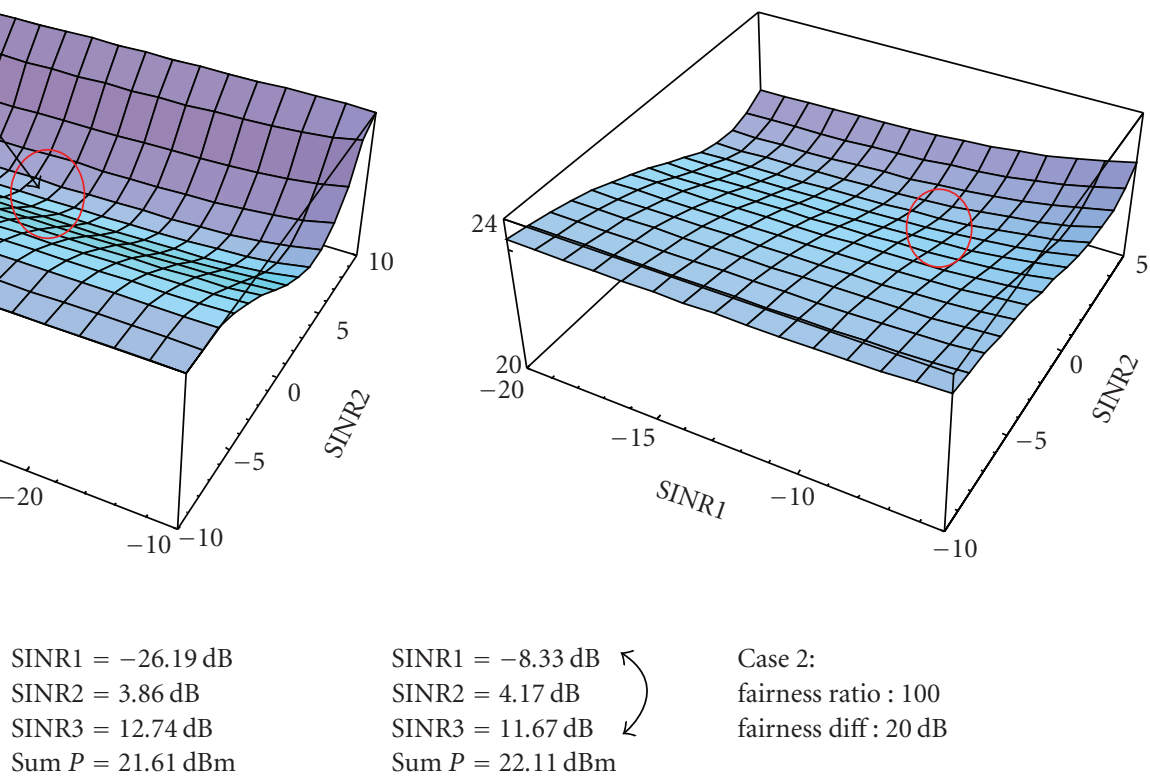

SINR1 $=-8.33 \mathrm{~dB} \quad$
SINR2 $=4.17 \mathrm{~dB}$
SINR3 $=11.67 \mathrm{~dB}$
Sum $P=22.11 \mathrm{dBm}$

Case 2:

fairness ratio : 100

fairness diff : $20 \mathrm{~dB}$

FIGURE 2: Sum power minimization. The figure shows the sum power as the function of SINR1 and SINR2 without fairness constraints (left) and for Case 2, when the ratios between the lowest and highest SINR must not be greater than 100. In this case the sum power is somewhat higher $(22.11 \mathrm{dBm})$ than without fairness constraint $(21.61 \mathrm{dBm})$.

fixed to 1 so that the SINR targets remain unchanged, thus reflecting the original algorithm in [13]; for problem (23), $\epsilon^{(t)}$ is tuned with a subgradient-like step until the power budget constraint is met with equality (point $\epsilon^{(t)}$ will not change anymore).

\section{Numerical Results}

In this section we consider a three-cell system, each of which is serving a single MS. In an OFDM cellular network, for example, this setting corresponds to the situation in which 


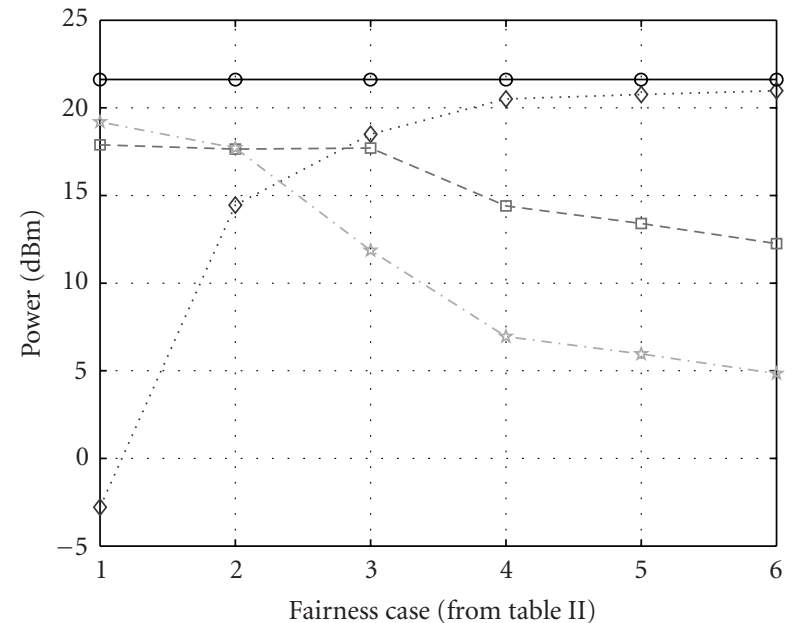

$$
\begin{array}{ll}
. \diamond \cdot \text { User } 1 & -\rightarrow-\text { User } 3 \\
-\boxminus-\text { User } 2 & 0 \text { Sum power }
\end{array}
$$

(a) The individual power levels for the six cases we study. The sum power is kept constant $(21.6 \mathrm{dBm})$, but the "distribution" of this power budget changes in the six fairness cases. Note that MS-3 needs to drastically increase its power at the cost of MS-1 and MS-2 to reach similar SINR

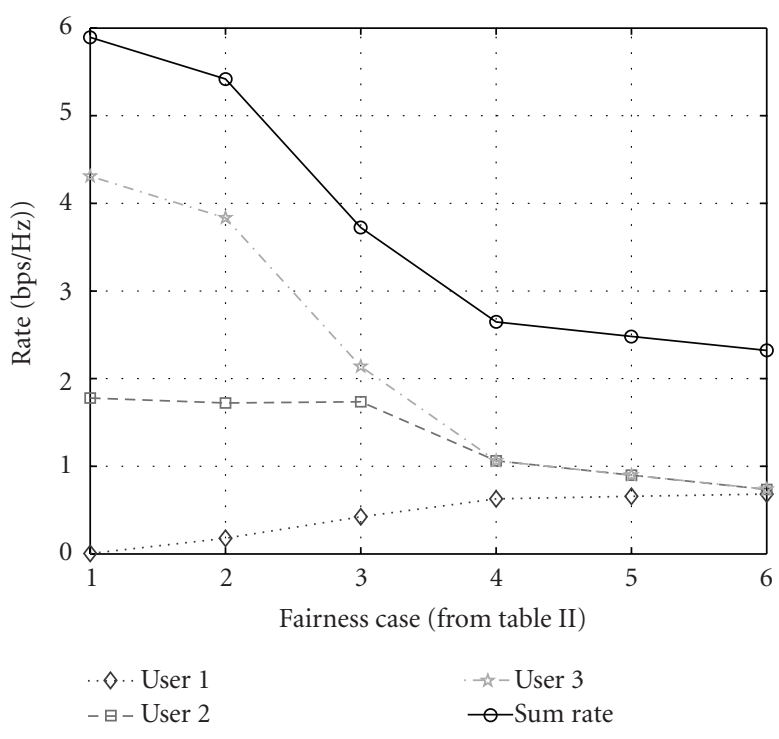

(b) The individual rates and the sum rate in the six fairness cases we study. The sum rate decreases as the SINR distribution becomes more fair. The Case 6 sum rate is only about $40 \%$ of the highest (no fairness) sum rate

FiguRE 3: Sum rate maximization subject to power budget and fairness constraints.

a single MS is served on an OFDM resource block and interference is caused by MS served in other cells (i.e., assuming perfect intracell orthogonality). The main parameters of this system are summarized in Table 1 . MS-1 is located at the cell edge, while MS-2 and MS-3 are close to their respective serving base stations. Table 2 reports six fairness ratios (in $\mathrm{dB}$ ) between the best and the worst SINR targets, reflecting increasing fairness constraints from unfair allocation (case 1) to almost egalitarian SINR allocation (case 6).

\subsection{Power Minimization under Rate and Fairness Constraints.} We have implemented the augmented Lagrangian penalty function method in Mathematica [21]. Figures 1-6 are obtained by generating the optimum SINR targets and power allocations for 1000 independent channel realizations. Figures 1 and 2 refer to the power minimization task when the sum rate target is kept constant 6 bps (normalized per $\mathrm{Hz}$ ) in the 3 cells.

Figure 1(b) illustrates the individual stream rates (hence implicitly the SINR targets) in each fairness case, Case 6 being the "most fair" SINR allocation at the expense of a total power increase at around $28.5 \mathrm{dBm}$ (Figure $1(\mathrm{a})$ ). Figure 1(b) confirms the fairness levels in the six cases in terms of the individual (per MS) rates. Figure 2 shows the sum power as a two-variable function of the SINR targets $\gamma_{1}^{\text {tgt }}$ and $\gamma_{2}^{\text {tgt }}$. The sum power attains its minimum $(21.6 \mathrm{dBm}$ $=145 \mathrm{~mW}$ ) when the SINR targets are set differently corresponding to an unfair rate allocation (Case 1). In Case 2 , there is a (loose) constraint on the ratio of SINR targets which can only be fulfilled at a somewhat increased total power level $(22.11 \mathrm{dBm})$.
7.2. Rate Maximization under Power and Fairness Constraints. Figures 3 and 4 show the results for the sum rate maximization task with the power budget set to $21.6 \mathrm{dBm}(145 \mathrm{~mW})$. In Figure 4 we see that the maximum sum rate is $6 \mathrm{bps}$; in other words this point is the same as the minimum power for the previous case. Similarly to the previous case, the unfair Case 1 provides the highest performance and enforcing more fair rate allocations reduces the achievable sum rate (Figures 3(a) and 3(b)). In Figure 3(a) we see that in Case 6, the cell edge user must take a lion share of the overall power budget. As Figure 3(b) clearly shows, the rate increase of the cell edge user is still much less than the rate loss of the cell center user, leading to an overall rate loss as compared with the unfair Case 1.

7.3. Accuracy and Computation Complexity. Next, we evaluate the technique based on the series convexifications described in Algorithm 1 and we compare it with the global optimization algorithm MAPEL. We consider a 5 -cell $1 \times 2$ MIMO network and we solve the sum-rate maximization without fairness constraints for a set of 50 channel realizations with users placed at fixed positions in the plane (the same in all experiments). The power budget is set to $21.6 \mathrm{dBm}$. Figure 5(a) compares the CDF of the computation time for MAPEL and the iterative convexification procedure, while Figure 5(b) exhibits the their output in terms of achieved sum rate. For MAPEL, we select an accuracy $\delta$ between 0.1 and 0.07 (we found experimentally that $\delta>0.1$ results in suboptimal points) and force the algorithm to stop after 2 hours. As we can see, the iterative convexification procedure converges to the optimal sum rate in only a couple of seconds while the computation time of MAPEL increases 

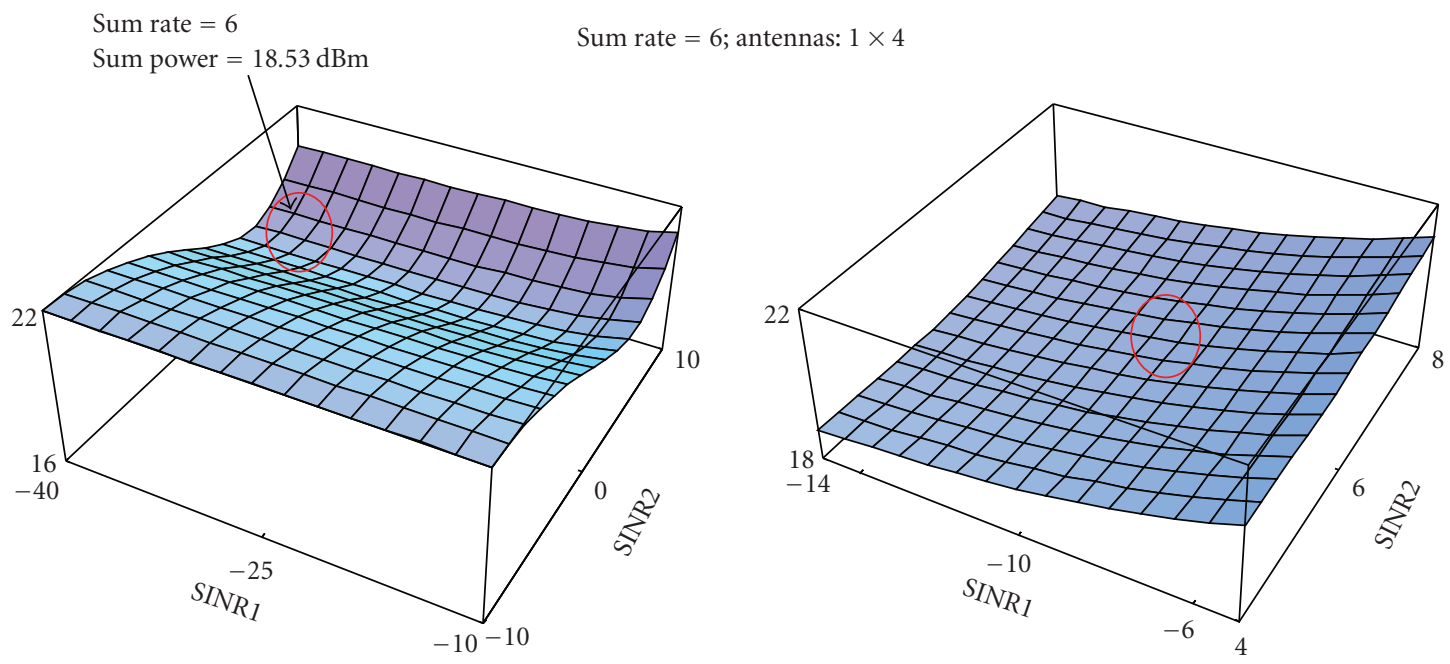

$$
\begin{aligned}
& \text { SINR1 }=-34.68 \mathrm{~dB} \\
& \text { SINR2 }=5.38 \mathrm{~dB} \\
& \text { SINR3 }=11.34 \mathrm{~dB} \\
& \text { Sum } P=18.53 \mathrm{dBm}
\end{aligned}
$$

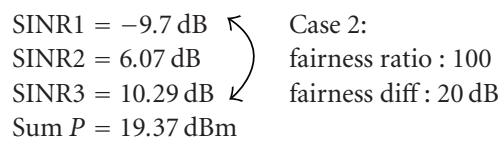

FIgURE 6: Sum power minimization for the $1 \times 4$ SIMO case. Compared with the $1 \times 2$ case (Figure 2), we notice that approximately half of the power is required to reach the same sum rate target $(18.53 \mathrm{dBm}=71.2 \mathrm{~mW})$.
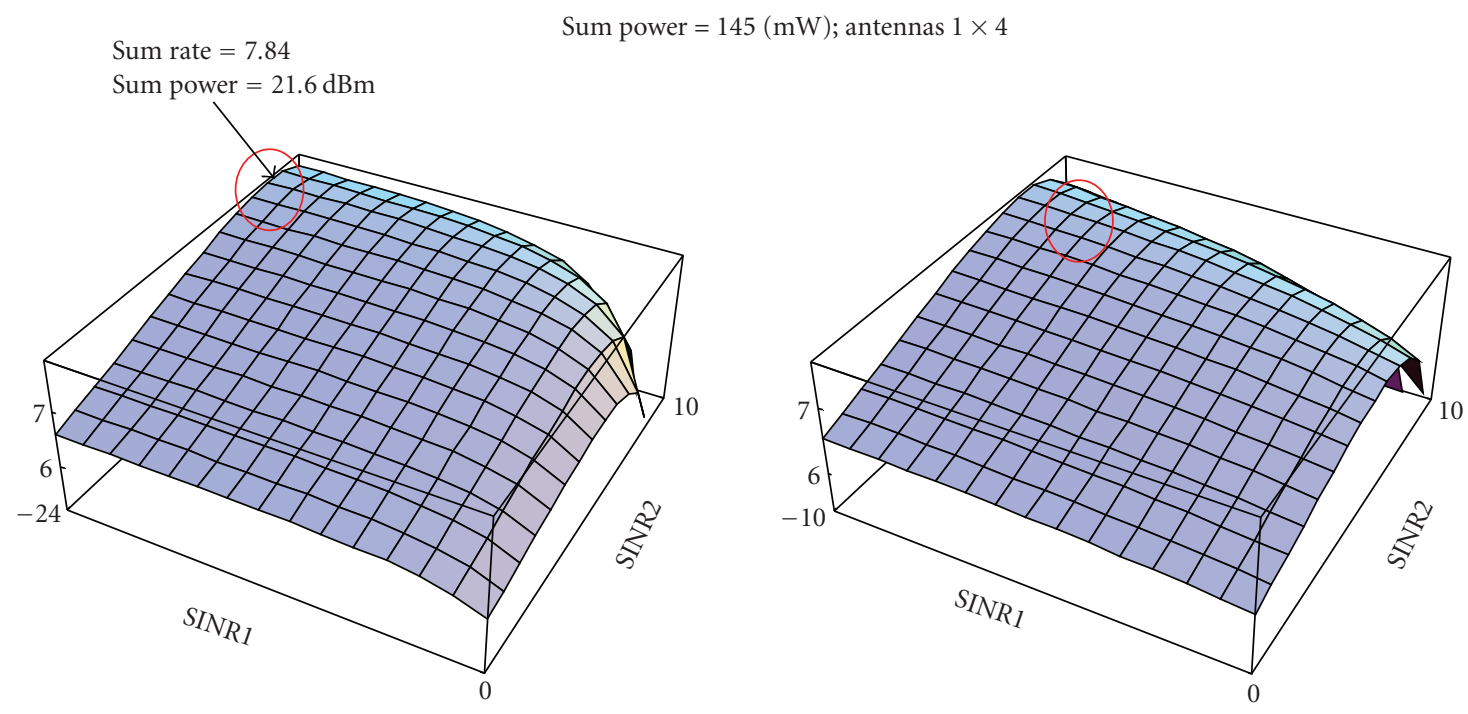

$$
\begin{aligned}
\text { SINR1 } & =-23.87 \mathrm{~dB} \\
\text { SINR2 } & =8.66 \mathrm{~dB} \\
\text { SINR3 } & =14.04 \mathrm{~dB} \\
\text { Sum } R & =7.84
\end{aligned}
$$

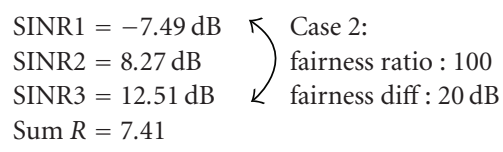

FIGURE 7: Capacity (sum rate) maximization with constant power budget (here $21.6 \mathrm{dBm} 145 \mathrm{~mW}$ ) for the $1 \times 4$ SIMO case. Compared with the $1 \times 2$ case (Figure 4 ), approximately $30 \%$ higher sum rate is reached in Case 1 .

the $1 \times 2$ SIMO case is sufficient to maintain similar rate performance. In the rate maximization case, it is possible to increase the $1 \times 2$ capacity with approximately $30 \%$ using the same power budget.
7.5. Precoding Gains. Following the structure of Section 6, we first combine our results on finding the optimal SINR targets for sum-power minimization with the iterative channel inversion power control algorithm of [13]. To this end, 


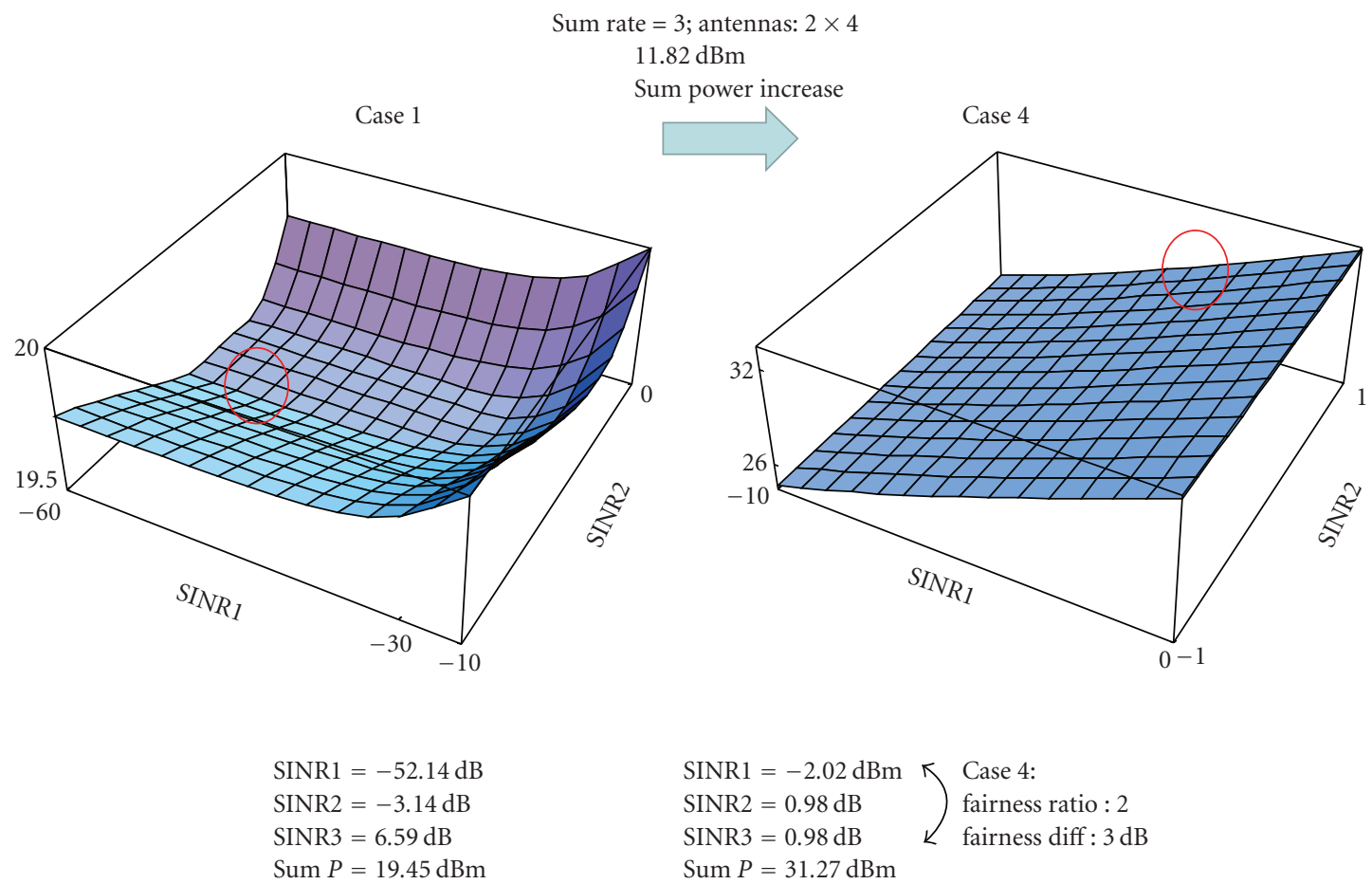

Figure 8: Power consumption without precoding in Case 1 and in Case 4. In Case 4, the lowest SINR must be at least half of the highest SINR MS. The point that satisfies this (and the capacity) constraint and minimizes the sum power is indicated on the right-hand side figure. With this constraint, the sum power is much higher than in the unconstraint case. Realistically, this fairness case is not feasible due to typical individual power limitations $(24 \mathrm{dBm})$.
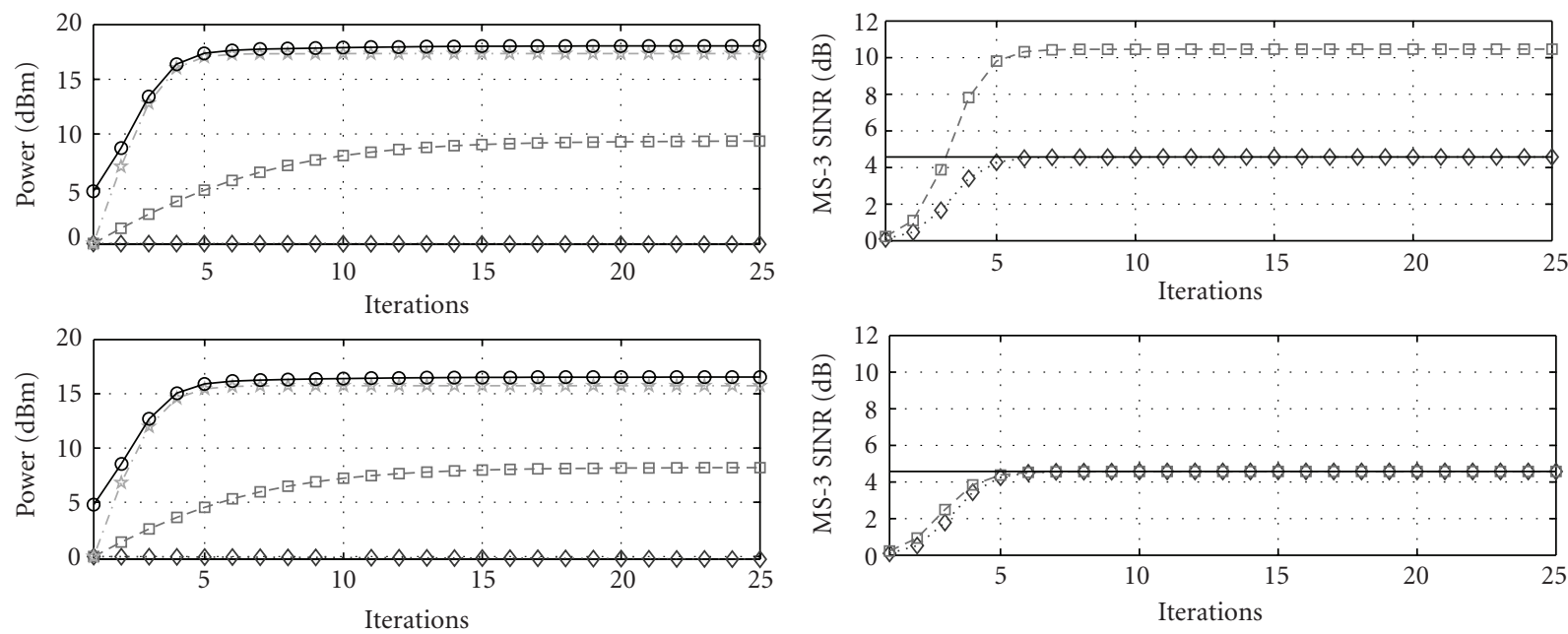

$$
\begin{aligned}
& \therefore \diamond \cdot \text { User } 1 \\
& \text { - th- User } 3 \\
& \text {-日-User } 2 \\
& \text { - }- \text { Sum power }
\end{aligned}
$$

(a) Power consumption without (upper) and with (lower) precoding in Case 1. Precoding has a significant impact on the minimum sum power that reaches the capacity target. The sum power is dominated by MS-3 whose power can be reduced by more than $30 \%$ due to precoding gains

FIGURE 9: The beneficial impact of transmission precoding in the sum power minimization problem (22) with optimal SINR target selection without fairness. 

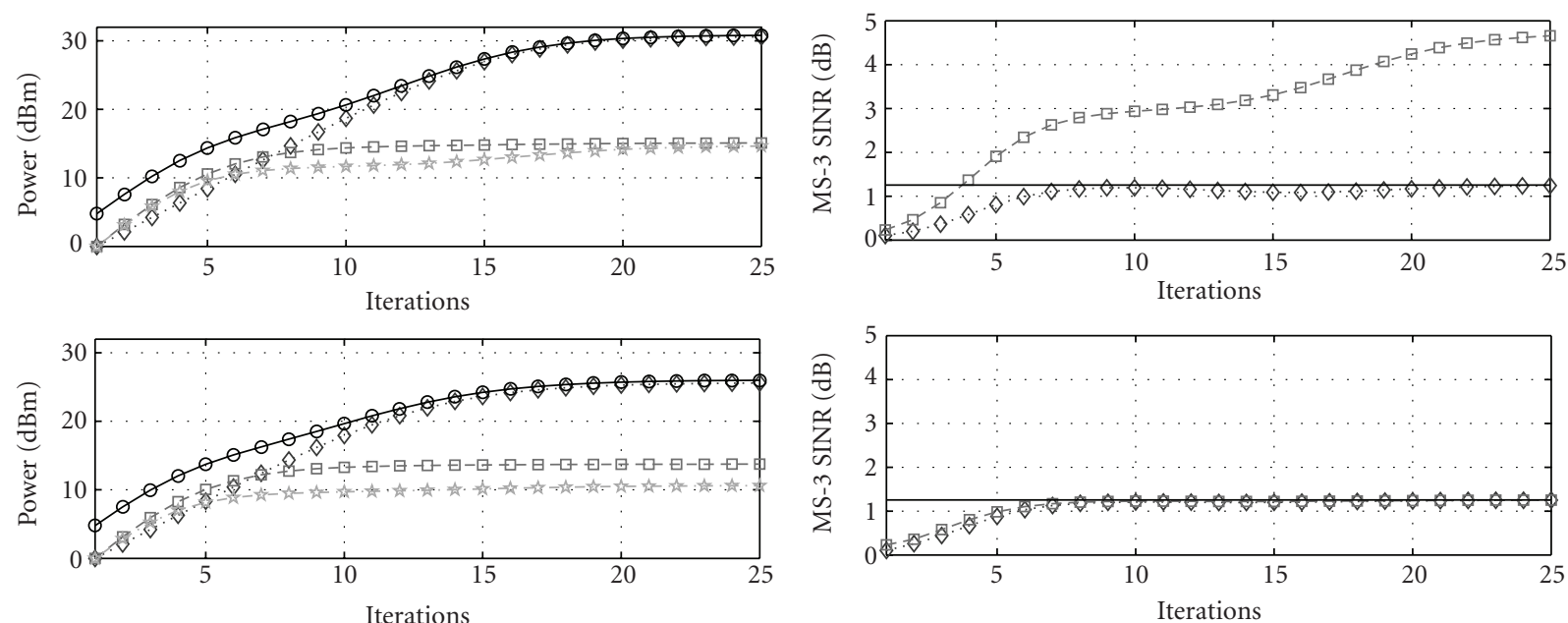

$$
\begin{array}{ll}
\diamond \text { User } 1 & \text { - User } 3 \\
-\boxminus-\text { User } 2 & - \text { Sum power }
\end{array}
$$

$$
\begin{aligned}
& \cdots \diamond \text { Stream } 1 \\
& -\boxminus-\text { Stream } 2 \\
& \longrightarrow \text { Target }
\end{aligned}
$$

(a) Power consumption without (upper) and with (lower) precoding in Case 4. Case 4 requires much higher sum power than Case 1 due to the fairness constraints. However, precoding makes even Case 4 feasible, due to the $66 \%$ power saving (see lower figure)

(b) SINR evolution for the two streams of MS-3 in Case 4. The figure is similar to Figure, but now precoding helps to reduce the higher SINR streams SINR from around $5(7 \mathrm{~dB})$ to $1.2(0.8 \mathrm{db})$

FIGURE 10: The beneficial impact of transmission precoding in the sum power minimization problem (22) with optimal SINR target selection with fairness.

we consider a three-cell $2 \times 4 \mathrm{MIMO}$ system with the input parameters of Table 1 and a capacity target of $3 \mathrm{bps} / \mathrm{Hz}$. For a given channel realization, we use the method of Section 4.1 to find the optimal SINR targets and use them as input values to the iterative channel inversion algorithm of [13] to find the associated power levels and precoding matrices for each mobile station.

The numerical results are shown in Figures 8-10. Figure 8 is obtained by the sum power minimization method of Section 4.1 without precoding. In Case 1, the required sum power is around $19.45 \mathrm{dBm}$, but in this case the SINR targets are drastically different (left-hand side). In Case 4, the imposed SINR fairness constraint dictates that the weakest SINR should be at least half of the strongest SINR, which leads to a dramatic sum power increase (right-hand side). In fact, this fairness case is not feasible in practical systems in which mobile stations are power limited to around $24 \mathrm{dBm}$.

Figure 9(a) shows the beneficial impact of using transmission precoding for the unconstrained case (Case 1). The horizontal axis shows the iteration steps of the iterative channel inversion algorithm, while the vertical axes show the individual and sum power levels. Here, the sum power is reduced more than $30 \%$ (on the linear scale) due to the power reduction of MS-3 from around $17 \mathrm{dBm}$ to around $15 \mathrm{dBm}$. This is an impressive precoding gain considering the fact the power levels of Case 1 are low even without precoding thanks to the optimal SINR target setting. This demonstrates a twofold gain of our approach compared to the alternatives in [13]: unlike a rough SINR target selection, we have shown that optimizing the targets is an efficient tool to enhance the throughput and control fairness in network MIMO systems. Furthermore, since the approximation used to derive the optimal SINR targets may lead to high power consumption, optimizing the precoding matrix $\mathbf{T}_{k}$ based on the given optimal SINR target allows to further reduce system sum-power.

In Figure 9(b) we follow the evolution of the per-stream SINR levels of MS-3 in Case 1 as determined by the iterative channel inversion algorithm. Recall from Figure 8 that the SINR target for MS-3 in Case 1 is 4.5686 (6.6 dB). With equal power allocation, the stream with higher SINR is significantly overallocated (upper figure). This waste of transmission power is eliminated with optimal precoding setting (lower figure) which allows for a lower transmission power of MS3. We also note that allocating less power for MS-3 reduces the interference caused to neighbor cells, which is a second contributing factor to an overall power decrease. Figure 10(a) compares the power levels without and with precoding for Case 4 . Here, the power saving due to optimal precoding is even more pronounced (from $30.8 \mathrm{dBm}$ to $25.6 \mathrm{dBm}$ which is $66 \%$ reduction on the linear scale) than in Case 1. By equalizing the SINRs of the two streams as shown in Figure 10(b), the transmission power of MS-3 is drastically reduced, thus making Case 4 becomes feasible.

Finally, we consider the effect of precoding optimization for the second class of problems where the SINR targets are selected to maximize the system throughput for a fixed power budget. Figure 11 illustrates the results for the same three-cell $2 \times 4$ MIMO system with the input parameters of Table I and a power budget $P_{\text {tot }}=21.6 \mathrm{dBm}$. From the 


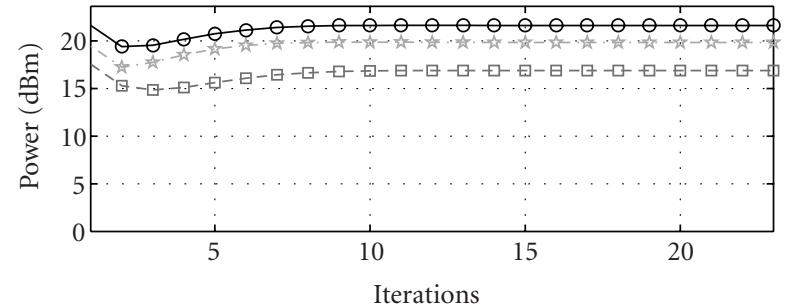

$$
\begin{aligned}
& \text { - }- \text { User } 2 \\
& \text { - User } 3 \\
& \text { - Sum power }
\end{aligned}
$$

(a)

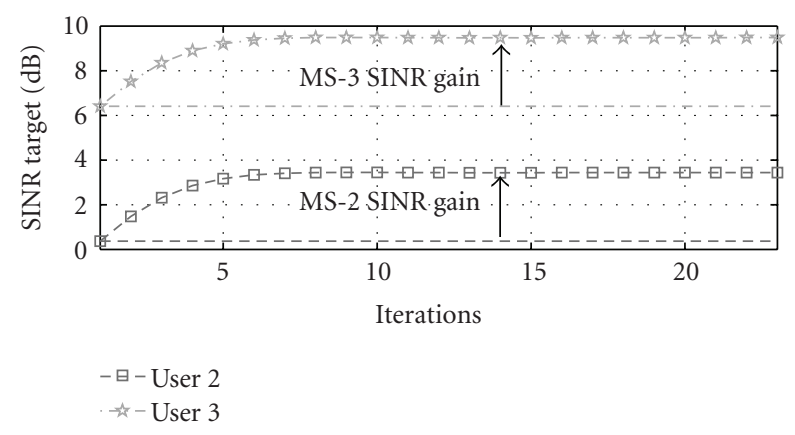

(b)

FIgure 11: Precoding gain in SINR target selection. The figure shows that for the $2 \times 4 \mathrm{MIMO}$ case with a power budget $21.6 \mathrm{dBM}$, optimizing the precoder matrices allows to increase the original (without precoding) optimal SINR targets for the same power budget. This gain is more than $3 \mathrm{~dB}$ per user.

TABLE 1: Input parameters of the 3-cell OFDMA system.

\begin{tabular}{ll}
\hline \multicolumn{2}{c}{ Input parameters } \\
\hline Inter Site Distance $[\mathrm{m}]$ & 500 \\
Distance of the MS- $i$ 's from & $0.45,0.15$, and 0.1 [ISD] \\
their serving BS $(i=1, \ldots, 3)$ & respectively \\
Path loss exponent & 3.07 \\
Shadow fading & Lognormal; st. dev: $10 \mathrm{~dB}$ \\
Fast fading model & Rayleigh flat \\
AWGN noise variance & $\sigma_{n}^{2}=0.01$ \\
Antenna configurations & $1 \times 2$ and $1 \times 4$ SIMO \\
\hline
\end{tabular}

TABLE 2: Fairness ratio between the best and worst SINR targets.

\begin{tabular}{lccccc}
\hline Case 1 & Case 2 & Case 3 & Case 4 & Case 5 & Case 6 \\
\hline- & $20 \mathrm{~dB}$ & $10 \mathrm{~dB}$ & $3 \mathrm{~dB}$ & $1.76 \mathrm{~dB}$ & $0.41 \mathrm{~dB}$ \\
\hline
\end{tabular}

initialization phase of Algorithm 2, the initial SINR targets derived with equal power allocation $\left(\mathbf{T}_{k}=\mathbf{I}_{N_{t}}\right.$ for all $\left.k\right)$ are $\boldsymbol{\Gamma}=\left[\begin{array}{ll}-47.4 & 0.36 \\ 6.41\end{array}\right] \mathrm{dB}$. By optimizing the precoding matrix, the same power budget allows to increase the SINR targets to the values $\Gamma=[-44.33 .449 .48] \mathrm{dB}$ with a throughput gain of roughly $42 \%$. Interestingly, this approach yields the same gain margin to all user's SINR target $(3.07 \mathrm{~dB}$ in this example) due to the multiplicative term $\epsilon^{(t)}$ used in (39).

Comparing Figures 2, 6, and 8, we observe that the optimal SINR targets are different for different antenna configurations. Our conjecture is that higher antenna systems, allowing for a higher overall capacity, may lead to greater differences in the SINR setting (and consequently a higher variation of the user bit rates) when no fairness constraint is imposed. The intuitive explanation is that there is a greater room for unequal SINR assignment in a system of higher capacity. Hence, in higher-order MIMO systems, dealing with fairness constraint may become increasingly important.

\section{Conclusions}

Tight coordination of network elements in cellular systems enables not only the introduction of network MIMO transmission and reception techniques but also the implementation of fast SINR target setting and tracking. We addressed the problem of optimally assigning SINR targets and transmission powers to mobile stations in tightly coordinated multicell spatial multiplexing systems. We considered two versions of the SINR target and power allocation problem: one that maximizes the sum rate subject to a power budget constraint, and another that minimizes the total power needed to meet a sum-rate target. Both formulations are constrained non-convex problems. We proposed a semianalytical solution via the augmented Lagrangian penalty function method and developed a fast numerical technique for the joint optimization of SINR targets and transmit powers. Numerical results demonstrated significant gains of the joint SINR target and power optimization depending on the degree of fairness imposed. In realistic network MIMO scenarios, our method displayed strong performance on a par with the globally optimal, but computationally very expensive, solution. We also showed how the transmission power needed to maintain a given capacity target can be reduced even further by also optimizing the precoding. A natural extension of this work is to design distributed schemes (such as that in [14]) for spatial multiplexing systems and to analyze their robustness against limited and inaccurate channel knowledge.

\section{Appendices}

\section{A. Derivations for the Linear MMSE}

For the sake of simplicity we rewrite the system model (3) as

$$
\mathbf{y}_{k}=\mathbf{A}_{k} \mathbf{x}_{k}+\mathbf{v}_{k}
$$

where $\mathbf{A}_{k}=\alpha_{k, k} \mathbf{H}_{k, k} \mathbf{T}_{k}$, the vector $\mathbf{x}_{k}$ is zero mean with covariance $\mathbf{R}_{x_{k}}=\mathbf{I}$, and $\mathbf{v}_{k}=\mathbf{z}_{k}+\mathbf{n}_{k}$ models the intercell interference plus noise with mean and covariance, respectively:

$$
\begin{aligned}
& \boldsymbol{\mu}_{v_{k}}=\mathbb{E}\left[\mathbf{v}_{k}\right]=\mathbb{E}\left[\mathbf{z}_{k}\right]+\mathbb{E}\left[\mathbf{n}_{k}\right]=0, \\
& \mathbf{R}_{v_{k}}=\mathbb{E}\left[\mathbf{v}_{k} \mathbf{v}_{k}^{\dagger}\right]=\mathbb{E}\left[\mathbf{z}_{k} \mathbf{z}_{k}^{\dagger}\right]+\mathbb{E}\left[\mathbf{n}_{k} \mathbf{n}_{k}^{\dagger}\right]=\mathbf{R}_{z_{k}}+\mathbf{R}_{n_{k}},
\end{aligned}
$$


where the intercell interference covariance matrix $\mathbf{R}_{z_{k}}$ is defined as in (4). Hence, $\mathbf{y}_{k}$ is zero-mean, and according to $[25,26]$ the linear MMSE receiver is given as

$$
\begin{aligned}
\mathbf{G}_{k}= & \mathbf{R}_{x_{k}} \mathbf{A}_{k}^{\dagger}\left(\mathbf{A}_{k} \mathbf{R}_{x_{k}} \mathbf{A}_{k}^{\dagger}+\mathbf{R}_{v_{k}}\right)^{-1}=\alpha_{k, k} \mathbf{T}_{k}^{\dagger} \mathbf{H}_{k, k}^{\dagger} \\
& \cdot\left(\alpha_{k, k}^{2} \mathbf{H}_{k k} \mathbf{T}_{k} \mathbf{T}_{k}^{\dagger} \mathbf{H}_{k, k}^{\dagger}+\sum_{j \neq k} \alpha_{k, j}^{2} \mathbf{H}_{k, j} \mathbf{T}_{j} \mathbf{T}_{j}^{\dagger} \mathbf{H}_{k, j}^{\dagger}+\sigma_{n}^{2} \mathbf{I}\right)^{-1} .
\end{aligned}
$$

Proposition 1 follows immediately by extracting $\alpha_{k, k}^{2}$.

A.1. Derivation of the MSE in Proposition 2. By applying the standard theory on linear MMSE computation to the model in (A.1), see, for example, [20, Chapter 12], the MMSE error covariance matrix for the $k$ th base station is

$$
\begin{aligned}
\mathbf{E}_{k}= & \mathbb{E}\left[\left(\widehat{\mathbf{x}}_{k}-\mathbf{x}_{k}\right)\left(\hat{\mathbf{x}}_{k}-\mathbf{x}_{k}\right)^{\dagger}\right] \\
= & \alpha_{k, k}^{2} \mathbf{G}_{k} \mathbf{H}_{k, k} \mathbf{T}_{k} \mathbf{T}_{k}^{\dagger} \mathbf{H}_{k, k}^{\dagger} \mathbf{G}_{k}^{\dagger}-2 \alpha_{k, k} \mathbf{G}_{k} \mathbf{H}_{k, k} \mathbf{T}_{k} \\
& +\mathbf{G}_{k} \mathbf{R}_{z_{k}} \mathbf{G}_{k}^{\dagger}+\mathbf{G}_{k} \mathbf{R}_{n_{k}} \mathbf{G}_{k}^{\dagger} \\
= & \left(\alpha_{k, k} \mathbf{G}_{k} \mathbf{H}_{k, k} \mathbf{T}_{k}-\mathbf{I}\right)\left(\alpha_{k, k} \mathbf{T}_{k}^{\dagger} \mathbf{H}_{k, k}^{\dagger} \mathbf{G}_{k}^{\dagger}-\mathbf{I}\right)+\mathbf{G}_{k} \mathbf{R}_{v_{k}} \mathbf{G}_{k}^{\dagger} .
\end{aligned}
$$

Finally, by replacing the expression of $\mathbf{G}_{k}$ in (A.3) into $\mathbf{E}_{k}$ and using similar techniques as in [17] we obtain

$$
\begin{aligned}
\mathbf{E}_{k}= & \mathbf{I}-\alpha_{k, k} \mathbf{T}_{k}^{\dagger} \mathbf{H}_{k, k}^{\dagger} \\
& \cdot\left(\alpha_{k, k}^{2} \mathbf{H}_{k, k} \mathbf{T}_{k} \mathbf{T}_{k}^{\dagger} \mathbf{H}_{k, k}^{\dagger}+\mathbf{R}_{v_{k}}\right)^{-1} \alpha_{k, k} \mathbf{H}_{k, k} \mathbf{T}_{k} \\
= & \left(I+\mathbf{T}_{k}^{\dagger} \mathbf{R}_{H_{k}} \mathbf{T}_{k}\right)^{-1},
\end{aligned}
$$

where $\mathbf{R}_{H_{k}}=\alpha_{k, k}^{2} \mathbf{H}_{k, k}^{\dagger} \mathbf{R}_{v_{k}}^{-1} \mathbf{H}_{k, k}$.

\section{B. Elements of the Sum Power Vector}

The parameters of (26) are as follows:

$$
\begin{aligned}
& M_{11}=\gamma_{1}^{\mathrm{tgt}} n_{1}\left(1+(1-\kappa) F_{23} F_{32} \gamma_{2}^{\mathrm{tgt}}\right), \\
& M_{12}=\gamma_{1}^{\mathrm{tgt}} \gamma_{2}^{\mathrm{tgt}} n_{2}\left(F_{12}-(1-\kappa) F_{13} F_{32}\right), \\
& M_{13}=-(1-\kappa)\left(F_{13}+F_{12} F_{23} \gamma_{2}^{\mathrm{tgt}}\right) \gamma_{1}^{\mathrm{tgt}} n_{3}, \\
& M_{21}=\gamma_{1}^{\mathrm{tgt}} \gamma_{2}^{\mathrm{tgt}} n_{1}\left(F_{21}-(1-\kappa) F_{23} F_{31}\right), \\
& M_{22}=\gamma_{2}^{\mathrm{tgt}} n_{2}\left(1+(1-\kappa) F_{13} F_{31} \gamma_{1}^{\mathrm{tgt}}\right), \\
& M_{23}=-(1-\kappa)\left(F_{23}+F_{13} F_{21} \gamma_{1}^{\mathrm{tgt}}\right) \gamma_{2}^{\mathrm{tgt}} n_{3}, \\
& M_{31}=-\gamma_{1}^{\mathrm{tgt}} n_{1}\left((1-\kappa)\left(F_{31}+F_{21} F_{32} \gamma_{2}^{\mathrm{tgt}}\right)\right), \\
& M_{32}=-\gamma_{2}^{\mathrm{tgt}} n_{2}\left((1-\kappa)\left(F_{32}+F_{12} F_{31} \gamma_{1}^{\mathrm{tgt}}\right)\right), \\
& M_{33}=-(1-\kappa)\left(1-F_{12} F_{21} \gamma_{1}^{\mathrm{tgt}} \gamma_{2}^{\mathrm{tgt}}\right) n_{3} ;
\end{aligned}
$$

where $\kappa=e^{c_{\mathrm{m}}-\log \left(1+\gamma_{1}^{\mathrm{tgt}}\right)-\log \left(1+\gamma_{2}^{\mathrm{tgt}}\right)}$, and

$$
\begin{aligned}
D_{p}= & 1-F_{12} F_{21} \gamma_{1}^{\mathrm{tgt}} \gamma_{2}^{\mathrm{tgt}}+(1-\kappa) \\
& \cdot\left(F_{31} \gamma_{1}^{\mathrm{tgt}}\left(F_{13}+F_{12} F_{23} \gamma_{2}^{\mathrm{tgt}}\right)+F_{32} \gamma_{2}^{\mathrm{tgt}}\left(F_{23}+F_{13} F_{21} \gamma_{1}^{\mathrm{tgt}}\right)\right) .
\end{aligned}
$$

\section{Proofs}

For ease of notation, we rewrite problem (34) as follows

$$
\begin{array}{ll}
\underset{\mathbf{p}, \mathbf{r}}{\operatorname{maximize}} & \sum_{k} r_{k} \\
\text { subject to } & r_{k} \leq W\left[\theta_{k}^{(t)} \log _{2}\left(\underline{\gamma}_{k}\left(e^{\widetilde{\mathbf{P}}}\right)\right)+\beta_{k}^{(t)}\right] \quad \forall k \\
& \sum_{k} e^{\widetilde{P}_{k}} \leq P_{\text {tot }},
\end{array}
$$

where we applied the approximation (33) to the link rate constraint and the change of variables $P_{k} \leftarrow e^{\widetilde{P}_{k}}$. In what follows, we use this formulation to prove our theoretical achievements since it easily maps back to the original nonconvex problem (30) and to the monotonic optimization (31).

C.1. Proof of Proposition 4. Similarly to [15, Lemma 4], we show that

$$
r_{k}^{(t)}=\widetilde{c}_{k}\left(\mathbf{p}^{(t)}, \theta_{k}^{(t)}, \beta_{k}^{(t)}\right) \leq c_{k}\left(\mathbf{p}^{(t)}\right)=\widetilde{c}_{k}\left(\mathbf{p}^{(t)}, \theta_{k}^{(t+1)}, \beta_{k}^{(t+1)}\right) .
$$

We prove the first relationship by contradiction. Assume that at the optimal solution of $\mathcal{P}^{(t)}$ the rate $r_{k}$ is strictly less than the approximate capacity. Then, we could increase $r_{k}$ (while keeping $\mathbf{p}$ fixed) until we achieve equality. This would improve the objective function; thus the solution was not optimal. The other two relations follow from the approximation (33). The rest of the proof follows analogously to [15, Lemma 4].

C.2. Proof of Theorem 5.1. Let $\left\{\mathbf{r}^{\star}, \widetilde{\mathbf{p}}^{\star}, \lambda^{\star}, \omega^{\star}\right\}$ denote the primal-dual optimal solution for the series of convex problems $\left\{P^{(t)}\right\}_{t}$. The associated KKT optimality conditions can be written as

$$
\begin{gathered}
\lambda_{k}^{\star} \theta_{k}^{\star}-\omega^{\star} e^{\widetilde{P}_{k}^{\star}}-\sum_{n \neq k} \lambda_{n}^{\star} \theta_{n}^{\star} \underline{\gamma}_{n}\left(e^{\tilde{\mathbf{p}}^{\star}}\right) \frac{G_{n k} e^{\widetilde{P}_{k}^{\star}}}{G_{n n} e^{\tilde{P}_{n}^{\star}}}=0, \\
1-\lambda_{k}^{\star}=0 .
\end{gathered}
$$

By Proposition 5, the problem sequence $\left\{P^{(t)}\right\}_{t}$ converges at which point the rate constraint becomes tight, that is,

$$
\widetilde{c}_{k}\left(\tilde{e}^{\widetilde{\mathbf{p}}^{(t)}}, \theta_{k}^{(t)}, \beta_{k}^{(t)}\right)=c_{k}\left(e^{\widetilde{\mathbf{p}}^{(t)}}\right)=\widetilde{c}_{k}\left(e^{\widetilde{\mathbf{p}}^{(t+1)}}, \theta_{k}^{(t+1)}, \beta_{k}^{(t+1)}\right) \quad \forall k .
$$


Therefore, by replacing $\mathbf{p}^{\star}=e^{\widetilde{\mathbf{p}}^{(t)}}$ and $\theta_{k}^{\star}=\underline{\gamma}_{k}\left(\mathbf{p}^{\star}\right) /(1+$ $\left.\underline{\gamma}_{k}\left(\mathbf{p}^{\star}\right)\right)$ into (C.3), the KKT conditions become

$$
\begin{gathered}
\lambda_{k}^{\star} \frac{\underline{\gamma}_{k}\left(\mathbf{p}^{\star}\right)}{1+\underline{\gamma}_{k}\left(\mathbf{p}^{\star}\right)}-\omega^{\star} P_{k}^{\star}-\sum_{n \neq k} \lambda_{n}^{\star} \frac{\underline{\gamma}_{n}^{2}\left(\mathbf{p}^{\star}\right)}{1+\underline{\gamma}_{n}\left(\mathbf{p}^{\star}\right)} \frac{G_{n k} P_{k}^{\star}}{G_{n n} P_{n}^{\star}}=0, \\
1-\lambda_{k}^{\star}=0
\end{gathered}
$$

It is easy to recognize that (C.6)-(C.7) coincides with the KKT optimality conditions of the original nonconvex problem (30). Thus, the problem series $\left\{P^{(t)}\right\}_{t}$ converges to a point $\left\{\mathbf{r}^{\star}, \mathbf{p}^{\star}\right\}$ that satisfies the KKT conditions of problem (30).

C.3. Proof of Theorem 5.2. Let $\boldsymbol{v}$ denote the Lagrange multipliers for the rate constraint in the monotonic optimization problem (31), and let $\left\{\tilde{\mathbf{r}}^{\star}, \widetilde{\mathbf{p}}^{\star}, \boldsymbol{\nu}^{\star}, \omega^{\star}\right\}$ denote the primaldual optimal solution. The associated KKT conditions can be written as

$$
\begin{gathered}
\frac{\nu_{k}^{\star}}{c_{k}^{\star}} \frac{\underline{\gamma}_{k}^{\star}}{1+\underline{\gamma}_{k}^{\star}}-\omega^{\star} e^{\widetilde{P}_{k}^{\star}}-\sum_{n \neq k} \frac{\nu_{n}^{\star}}{c_{n}^{\star}} \frac{\underline{\gamma}_{n}^{\star}}{1+\underline{\gamma}_{n}^{\star}} \frac{G_{n k} e^{\widetilde{P}_{k}^{\star}}}{G_{n n} e^{\widetilde{P}_{n}^{\star}}}=0, \\
e^{\tilde{r}_{k}^{\star}}-v_{k}^{\star}=0,
\end{gathered}
$$

where $\underline{\gamma}_{k}^{\star} \triangleq \underline{\gamma}_{k}\left(e^{\tilde{\mathbf{p}}^{\star}}\right)$ and $c_{k}^{\star} \triangleq c_{k}\left(e^{\tilde{\mathbf{p}}^{\star}}\right)$. Moreover, (C.9) can be expressed equivalently as follows:

$$
e^{r_{k}^{\star}}-v_{k}^{\star}=0 \Longleftrightarrow r_{k}^{\star}-v_{k}^{\star}=0 \Longleftrightarrow r_{k}^{\star}\left(1-\frac{v_{k}^{\star}}{r_{k}^{\star}}\right)=0 .
$$

Let now $\left\{\hat{\mathbf{r}}^{\star}, \hat{\mathbf{p}}^{\star}, \lambda^{\star}, \omega^{\star}\right\}$ denote the optimal solution of the problem series $\left\{\mathcal{P}^{(t)}\right\}_{t}$, along with the approximation vectors $\left\{\boldsymbol{\theta}^{\star}, \boldsymbol{\beta}^{\star}\right\}$. By Theorem 5.1 , this solution satisfies the KKT optimality conditions (C.7), and by Proposition 5 all constraints are active and the approximation becomes exact, that is,

$$
\hat{r}_{k}^{\star}=\widetilde{c}_{k}\left(\hat{\mathbf{p}}^{\star}, \boldsymbol{\theta}^{\star}, \boldsymbol{\beta}^{\star}\right)=c_{k}\left(\hat{\mathbf{p}}^{\star}\right) \quad \forall k
$$

By constructing an auxiliary set of optimal dual variables as

$$
\nu_{k}^{\star}=\lambda_{k}^{\star} c_{k}\left(\hat{\mathbf{p}}^{\star}\right)=\lambda_{k}^{\star} \hat{r}_{k}^{\star} \quad \forall k,
$$

the proof follows immediately by replacing $\lambda_{k}^{\star}=v_{k}^{\star} / c_{k}\left(\hat{\mathbf{p}}^{\star}\right)$ and $\lambda_{k}^{\star}=v_{k}^{\star} / \hat{r}_{k}^{\star}$ in (C.6)-(C.7), respectively.

\section{Acknowledgments}

This work was part of the SERAN project and in part supported by VINNOVA. The authors thank Dr. Claes Tidestav, Dr. Mikael Prytz, and Mats Blomgren, from Ericsson Research, for their valuable comments throughout this work. They also thank the anonymous reviewers for the valuable comments that improved the contents and the presentation of the paper.

\section{References}

[1] M. K. Karakayali, G. J. Foschini, and R. A. Valenzuela, "Network coordination for spectrally efficient communications in cellular systems," IEEE Wireless Communications, vol. 13, no. 4, pp. 56-61, 2006.

[2] D. Samardzija, H. Huang, R. Valenzuela, and T. Sizer, "An experimental downlink multiuser MIMO system with distributed and coherently-coordinated transmit antennas," in Proceedings of IEEE International Conference on Communications (ICC '07), pp. 5365-5370, Glasgow, UK, June 2007.

[3] T. Tamaki, K. Seong, and J. M. Cioffi, "Downlink MIMO systems using cooperation among base stations in a slow fading channel," in Proceedings of IEEE International Conference on Communications (ICC'07), pp. 4728-4733, Glasgow, UK, June 2007.

[4] T.-D. Nguyen, O. Berder, and O. Sentieys, "Impact of transmission synchronization error and cooperative reception techniques on the performance of cooperative MIMO systems," in Proceedings of IEEE International Conference on Communications (ICC '08), pp. 4601-4605, Beijing, China, May 2008.

[5] H. Zhang, G.-S. Kuo, and T. M. Bohnert, "Cooperative diversity for virtual MIMO system in the presence of spatial correlated fading model," in Proceedings of the 68th IEEE Vehicular Technology Conference (VTC '08), Calgary, Canada, September 2008.

[6] P. Komulainen and M. Boldi, Eds., "Initial Report on Advanced Multiple Antenna Systems," Deliverable of the Wireless World Initiative New Radio - WINNER+, Januray 2009.

[7] "Deployment Aspects," Third Generation Partnership Project (3GPP), June, 2007.

[8] W. Choi and J. G. Andrews, "The capacity gain from base station cooperative scheduling in a MIMO DPC cellular system," in Proceedings of IEEE International Symposium on Information Theory (ISIT '06), pp. 1224-1228, Seattle, Wash, USA, July 2006.

[9] H. Kim and R. M. Buehrer, "Power allocation strategies in cooperative MIMO networks," in Proceedings of IEEE Wireless Communications and Networking Conference (WCNC'06), vol. 3, pp. 1675-1680, Las Vegas, Nev, USA, April 2006.

[10] M. Nokleby, A. L. Swindlehurst, Y. Rong, and Y. Hua, "Cooperative power scheduling for wireless MIMO networks," in Proceedings of the 50th Annual IEEE Global Telecommunications Conference (GLOBECOM '07), pp. 2982-2986, Washington, DC, USA, November 2007.

[11] G. Li and H. Liu, "Downlink radio resource allocation for multi-cell OFDMA system," IEEE Transactions on Wireless Communications, vol. 5, no. 12, pp. 3451-3459, 2006.

[12] C. Koutsimanis and G. Fodor, "A dynamic resource allocation scheme for guaranteed bit rate services in OFDMA networks," in Proceedings of the IEEE International Conference on Communications (ICC '08), pp. 2524-2530, Beijing, China, May 2008.

[13] R. Chen, J. G. Andrews, R. W. Heath Jr., and A. Ghosh, "Uplink power control in multi-cell spatial multiplexing wireless systems," IEEE Transactions on Wireless Communications, vol. 6, no. 7, pp. 2700-2711, 2007.

[14] P. Hande, S. Rangan, M. Chiang, and X. Wu, "Distributed uplink power control for optimal SIR assignment in cellular data networks," IEEE/ACM Transactions on Networking, vol. 16, no. 6, pp. 1420-1433, 2008. 
[15] J. Papandriopoulos, S. Dey, and J. Evans, "Optimal and distributed protocols for cross-layer design of physical and transport layers in MANETs," IEEE/ACM Transactions on Networking, vol. 16, no. 6, pp. 1392-1405, 2008.

[16] P. Soldati and M. Johansson, "Reducing signaling and respecting timescales in cross-layer protocols design for wireless networks," in Proceedings of the IEEE Global Telecommunications Conference (GLOBECOM '09), Honolulu, Hawaii, USA, December 2009.

[17] D. P. Palomar, "Convex primal decomposition for multicarrier linear MIMO transceivers," IEEE Transactions on Signal Processing, vol. 53, no. 12, pp. 4661-4674, 2005.

[18] H. Dai, A. F. Molisch, and H. V. Poor, "Downlink capacity of interference-limited MIMO systems with joint detection," IEEE Transactions on Wireless Communications, vol. 3, no. 2, pp. 442-453, 2004.

[19] J. G. Andrews, W. Choi, and R. W. Heath Jr., "Overcoming interference in spatial multiplexing mimo cellular networks," IEEE Wireless Communications, vol. 14, no. 6, pp. 95-104, 2007.

[20] S. M. Kay, Fundamentals of Statistical Signal Processing: Estimation Theory, vol. 1 of Prentice Hall Signal Processing Series, Prentice-Hall, Englewood Cliffs, NJ, USA, 1993.

[21] M. A. Bhatti, Practical Optimization Methods with Mathematica Applications, Springer, Berlin, Germany, 2000.

[22] L. P. Qian, Y. J. Zhang, and J. Huang, "MAPEL: achieving global optimality for a non-convex wireless power control problem," IEEE Transactions on Wireless Communications, vol. 8, no. 3, pp. 1553-1563, 2009.

[23] J. Papandriopoulos and J. S. Evans, "SCALE: a low-complexity distributed protocol for spectrum balancing in multiuser DSL networks," IEEE Transactions on Information Theory, vol. 55, no. 8, pp. 3711-3724, 2009.

[24] R. A. Horn and C. R. Johnson, Matrix Analysis, Cambridge University Press, Cambridge, UK, 1985.

[25] A. Paulraj, Course Notes, EE 492m, Stanford University, Stanford, Calif, USA, 2002.

[26] S. Boyd, Course Notes, EE 363, Stanford University, Stanford, Calif, USA, 2003. 

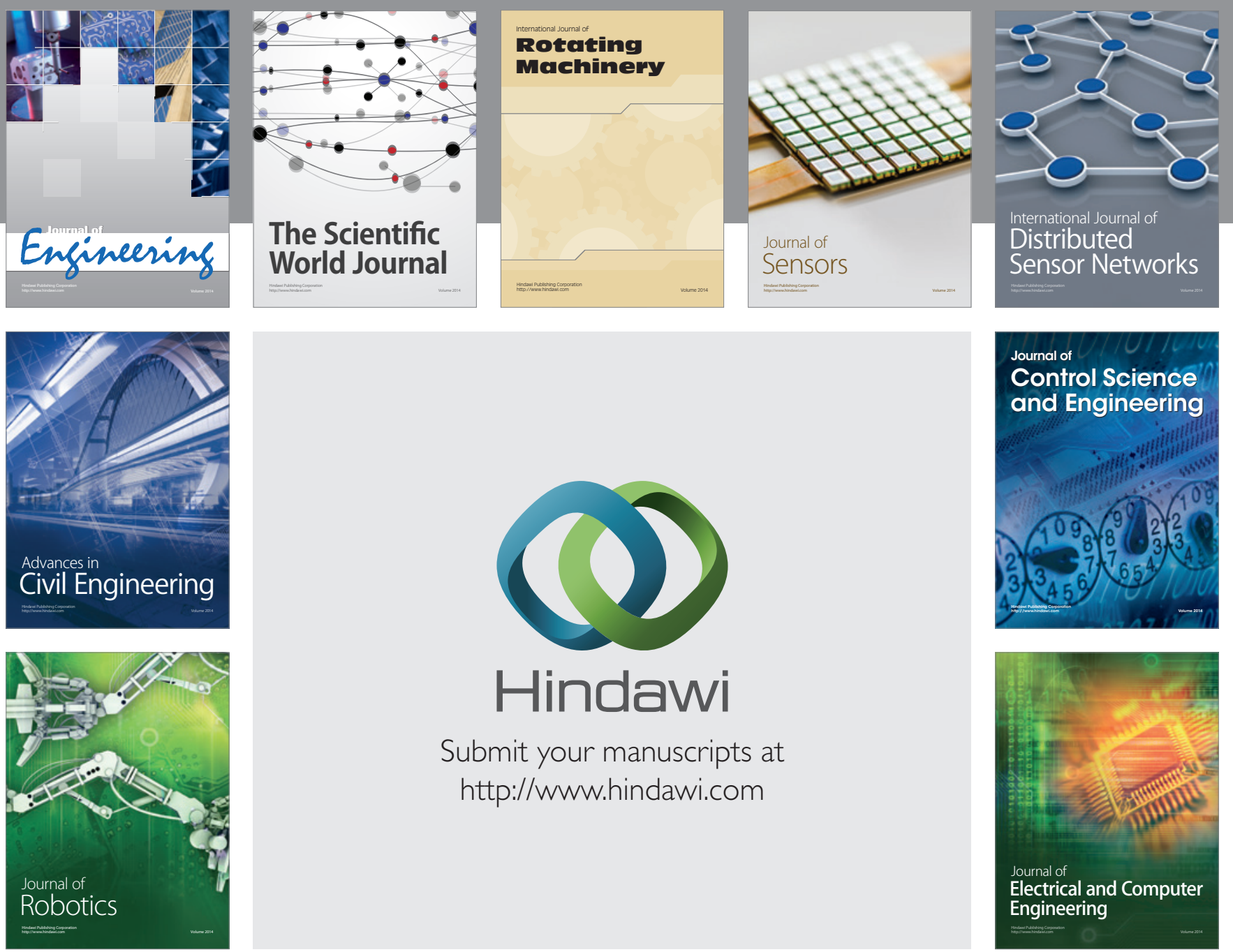

Submit your manuscripts at

http://www.hindawi.com
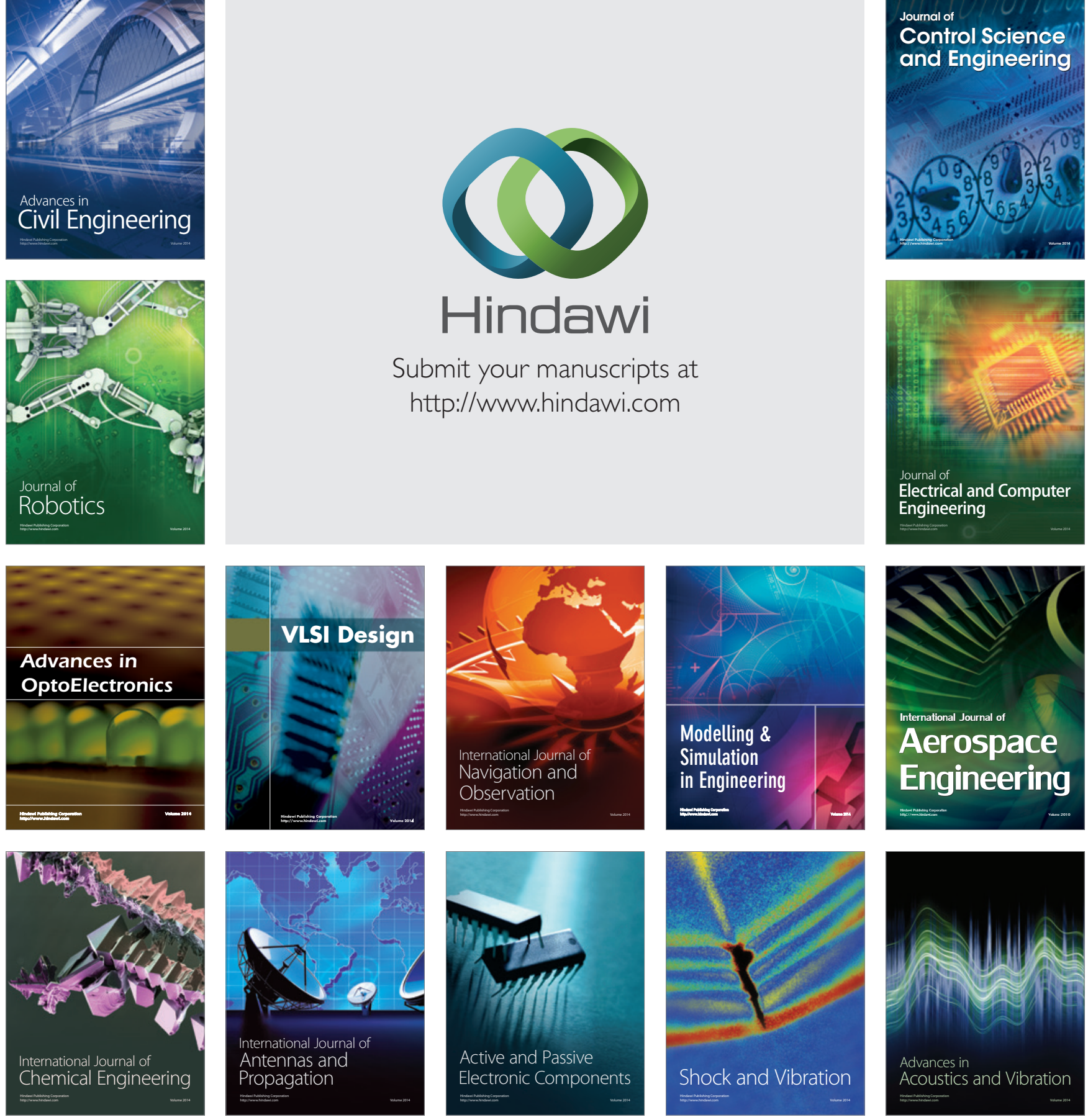